\title{
3D/4D-printed Bending-type Soft Pneumatic Actuators: Fabrication, Modelling, and Control
}

\begin{abstract}
This article reviews soft pneumatic actuators (SPAs) that are manufactured entirely via additive manufacturing methods. These actuators are known as four-dimensional (4D)-printed SPAs and can generate bending motions in response to either pressurized or vacuum (negative pressure) air stimulus after fabrication. They are characterized by geometrical and material factors that determine their motion trajectory, and the force they exert on manipulated soft objects in delicate applications such as food handling and non-invasive surgery. Here, we review various $3 \mathrm{D}$ printers and materials used for the fabrication of the pressurized air bendingtype SPAs. The reported approaches for modeling and control of these actuators are presented and compared. General discussions, as well as future directions and challenges of these actuators, are given.
\end{abstract}

Keywords: soft pneumatic actuators; soft robots; 3D printing; 4D printing

\section{Contents}

1. Introduction

2. Fabrication of 4D-printed bending-type soft pneumatic actuators

3. Modeling of 4D-printed bending-type soft pneumatic actuators

3.1. Analytical kinematic model

3.2. Geometrical parameters effects on the bending angle of soft pneumatic actuators

3.3. Analytical blocking/tip force model

4. Finite Element Analysis of 3D/4D-Printed Bending-type Soft Pneumatic Actuators

5. Control of 4D-printed Bending-type Soft Pneumatic Actuators

5.1. Integration of sensors

5.2. Data-driven machine learning modeling and control

6. Discussions, Challenges, and Future Directions

7. Conclusion

References

\section{Introduction}

Actuators are the devices that cause motion, which could be linear, rotary, or their combination, to move joints and body of the robot. There are several different types of actuators in soft robotics, which are classified in terms of their source of energy and structural materials, such as pneumatic (Yap, Ng, and Yeow 2016), hydraulic (Cacucciolo et al. 2019), liquid crystal (Roach et al. 2018), liquid metal (He, Zhou, et al. 2018), and ionic polymer actuators 
(Zolfagharian, Kouzani, Khoo, Moghadam, et al. 2016). The soft actuators exert force upon mechanical changes in response to the various stimuli, such as pressure, electricity, heat, humidity, and light (Zolfagharian, Kouzani, Khoo, Gibson, et al. 2016).

Four-dimensional (4D) printing has quickly found applications in soft robots and actuators fabricated from soft materials, including polymers, hydrogels, and liquid crystals, which respond to external stimulation such as pneumatic, electrical, $\mathrm{pH}$, and heat (Tibbits 2014; Bodaghi, Damanpack, and Liao 2017, 2016; Khoo et al. 2015; Leist and Zhou 2016). The two major practical applications of 4D printing are the 3D-printed soft pneumatic actuators (SPAs) and soft pneumatic robots producing mechanical motions in response to pressurized or vacuum (negative pressure) air stimulus (Tawk, Gillett, et al. 2019). In comparison to hydraulic actuators, the pneumatic actuators are simpler and cleaner due to operation by air, and notably deliver more compliance because of the lower density and viscosity thus making it possible to be used in applications which require the working fluid to travel relatively fast through lumens with small diameters (Davis 2018). These products have been ideally suited to 4D printing due to ease of fabrication by using elastomeric materials. The 3D/4D-printed SPAs generated sophisticated motions (highlighted in Fig. 1) in response to simple pressurized air input (Walker et al. 2020), including bending (Ge et al. 2018), rotating (Ainla et al. 2017), twisting (Connolly, Walsh, and Bertoldi 2017), jumping (Bartlett et al. 2015), rolling (Robertson and Paik 2017), and their combinations ( $\mathrm{Hu}, \mathrm{Li}$, and Alici 2018) with effective control capability. Desirable properties such as flexibility, lightweight, large amplitude, and repeatability of motion, safety in a human-robot interactive environment, inexpensiveness and ease of fabrication, associated with these soft actuators could not be realized by using traditional robots. These unique attributes offered their implementations in industrial applications (highlighted in Fig. 2) such as fruit harvesting (Wang, Torigoe, and Hirai 2017), food packaging (Wang, Zhu, et al. 2017; Wang, Chathuranga, and Hirai 2016; Yamanaka et al. 2020), space exploration (Kisner, Szigeti, and Leonardo 2018) and non-invasive surgery (Liang, Sun, and Ren 2016).

In this study, we focus on bending-type SPAs operating with pressurized air stimulus fabricated via different 3D printing technologies and materials. Fabrication of the 3D-printed SPAs could be conducted through various elastomeric materials and resins and 3D printing technologies. Thus far, the 3D-printed SPAs have been fabricated by Multi Jet Fusion (MJF) (Lau 2019; Zhu et al. 2018), fused deposition modelling (FDM), direct ink writing (DIW) (Plott and Shih 2017; Schaffner et al. 2018), selective laser sintering (SLS) (Scharff et al. 2017), stereolithography (SLA) (Peele et al. 2015), and digital light processing (DLP) (Ge et al. 2018) which are discussed in detail.

The kinematics and dynamics modeling of the 3D-printed SPAs are different from the conventional rigid manipulators due to the properties of soft materials used in their fabrication (Webster III and Jones 2010; Zolfagharian, Kaynak, and Kouzani 2019). Therefore, recent studies have proposed new static, kinematic, and dynamic models for predicting the 3D-printed SPAs behavior, taking their body morphology, and the pressure system characteristics into consideration. Step-wise methods, including the analytical and finite element analysis (FEA) approaches, are reviewed and addressed here to demonstrate the relationship among the input pressure, geometry, material characteristics, and the bending curvature of 3D-printed SPAs. 


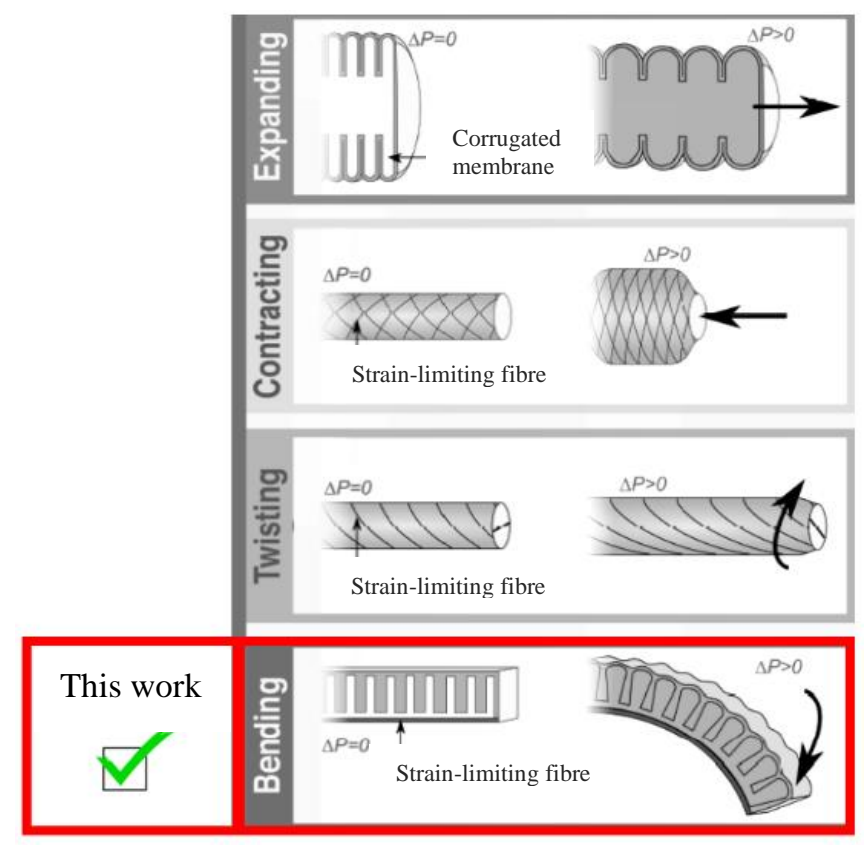

Fig. 1. Various types of 3D/4D-printed SPAs, reprinted from (Gorissen et al. 2017) with the permission of John Wiley and Sons.

The SPAs were initially developed because their passive compliance was desirable enough to tolerate the uncertainties that emerged while manipulating the delicate objects with various shapes and textures without the need for costly sensors and controllers. However, it has so far been challenging to accurately carry out pick and place tasks, especially on soft objects with variable shapes and orientations, without feedback information (Elgeneidy, Lohse, and Jackson 2018). Thus, equipping them with some level of sensing capabilities would lead to more controllable SPAs with enhanced functionality and a wider range of applications involving more complex manipulation tasks. The control of 3D/4D-printed SPAs is highly reliant on the integrated flexible electronics interfaces (Tan, Tran, and Chua 2016) and sensors (Zolfagharian, Kaynak, and Kouzani 2019). These components provide the necessary feedback information to help with the decision making of the manipulator without interfering with the compliance and morphology of a soft manipulator body (Zolfagharian et al. 2020). A broad range of chemical and biological sensors, which allow for more accurate and delicate manipulations, are also discussed in this study. Finally, appropriate control approaches for 3D/4D-printed bending-type SPAs are presented.

In light of the above discussion, the remainder of the paper is organized as follows: Section 2, is an overview of the fabrication of 3D/4D-printed bending soft pneumatic actuators. Section 3 , presents analytical modeling for estimating the bending behavior of these actuators. Section 4, reviews different energy function methods in FEA of the 3D/4D-printed SPAs based on the materials used in their fabrications. Section 5, investigates control-based 3D/4D-printed SPAs. Section 6, discusses the current challenges of these actuators and suggests innovative research possibilities. Section 7 concludes the paper. 


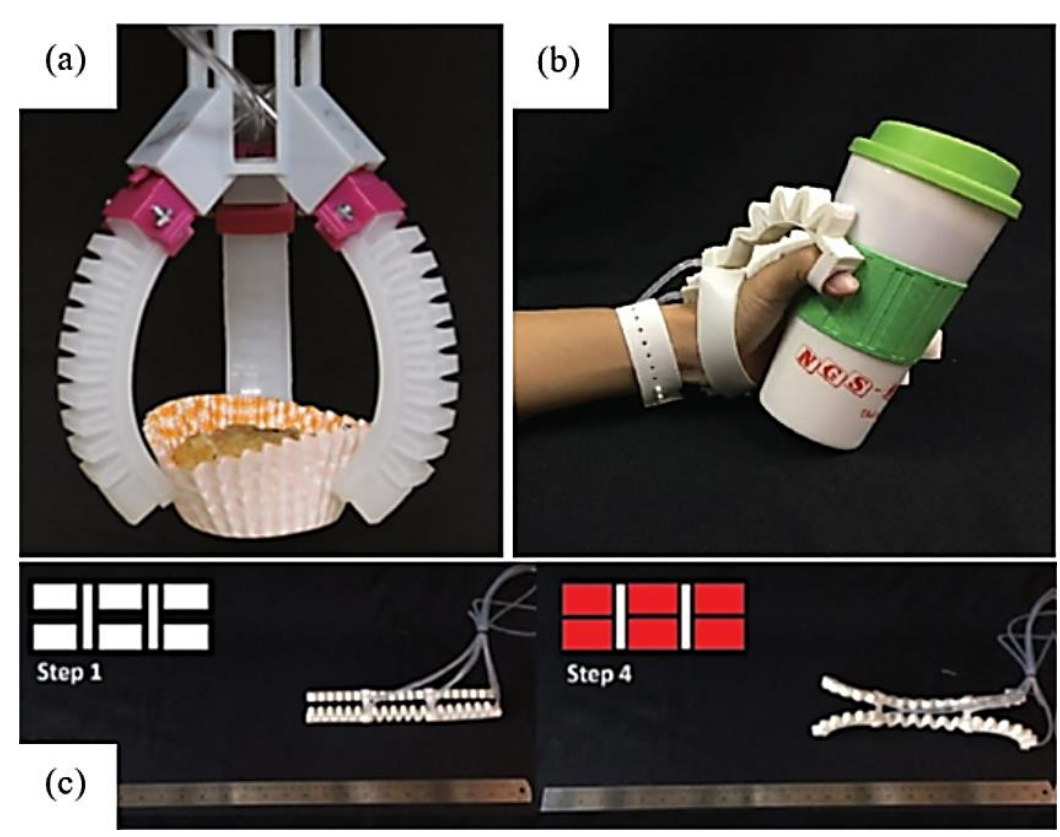

Fig. 2. Various applications of 3D/4D-printed SPAs; (a) food handling, reprinted from (Wang, Zhu, et al. 2017) with permission of Springer Nature open-access article distributed under the terms of the Creative Commons CC BY license; (b) rehabilitation, reprinted from (Keong and Hua 2018) with permission of John Wiley and Sons; (c) locomotion soft robot, reprinted from (Keong and Hua 2018) with permission of John Wiley and Sons.

\section{Fabrication of 4D-printed bending-type soft pneumatic actuators}

There have been extensive efforts on developing and testing soft pneumatic robots and actuators, the majority of which made by silicone elastomers, using molding and casting techniques (Batsuren and Yun 2019; Gorissen et al. 2017; Polygerinos et al. 2015; Mosadegh et al. 2014; Gorissen et al. 2014; Tolley et al. 2014; Sun, Song, and Paik 2013). Yet, there are some challenges associated with these methods including, intensive post-processing and labor, limited flexibility in design, and lack of repeatability in custom-made manufacturing with finely detailed features. It has been evident that the complexity involved in the structure of the soft actuators prolonged the fabrication time and complicated the procedure. However, additive manufacturing has been recently introduced as a promising approach for the fabrication of complex soft pneumatic robots and actuators with high resolution and repeatability. 3D printing has enabled producing elastomeric and thermoplastic materials under certain conditions. Different 3D printing technologies (highlighted in Fig. 3) have been employed for the fabrication of the current bending-type SPAs. 


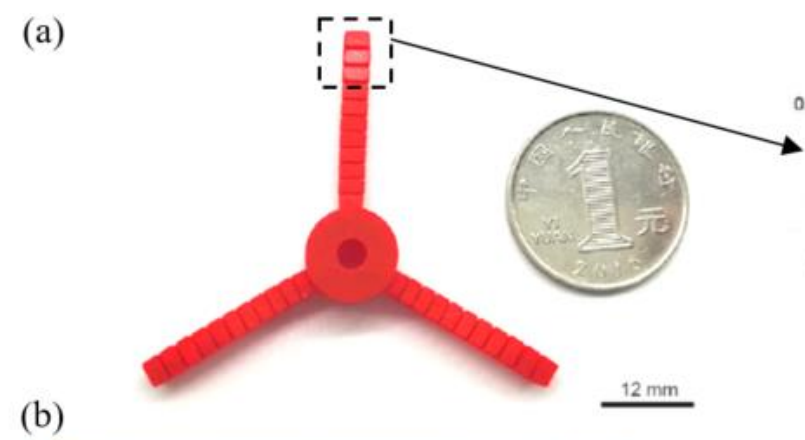

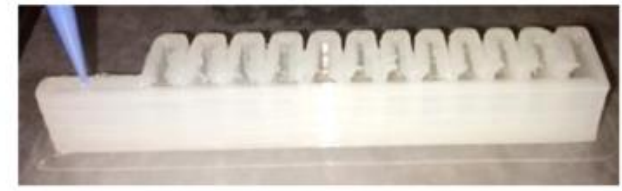

(c)

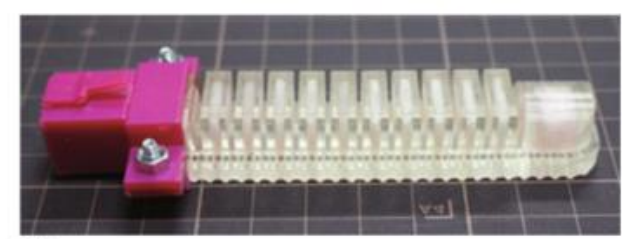

(d)

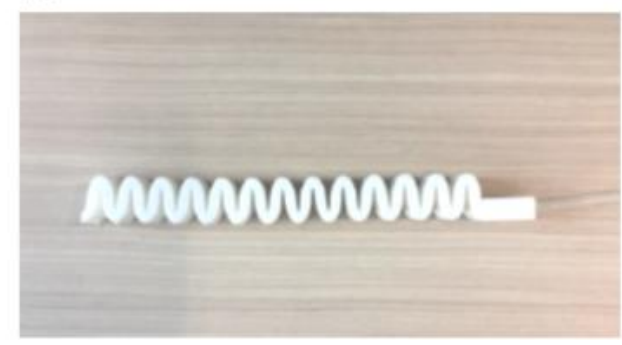

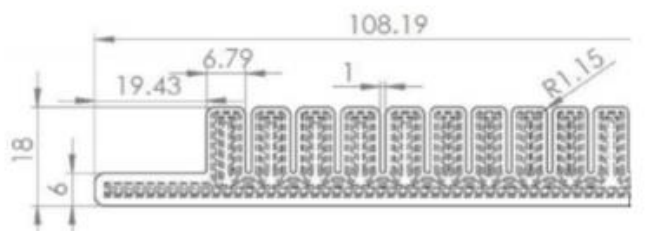
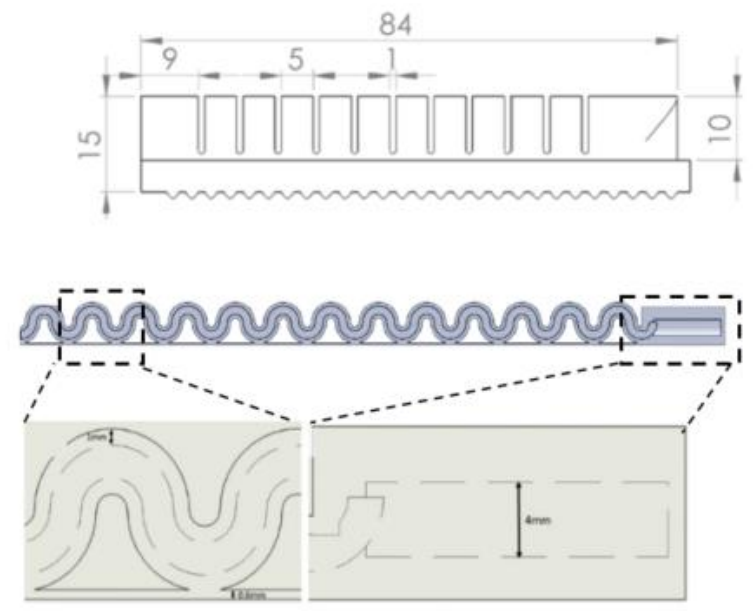

Fig. 3. 3D/4D-printed SPAs fabricated using different 3D printing methods (a) DLP, reprinted from (Ge et al. 2018) with permission of Elsevier; (b) DIW, reprinted from (Plott and Shih 2017) with permission of Elsevier; (c) Polyjet, reprinted from (Wang, Zhu, et al. 2017) with permission of Springer Nature under the terms of the Creative Commons CC BY license; (d) FDM, reprinted from (Keong and Hua 2018) with permission of John Wiley and Sons.

The DIW is an extrusion-based 3D printing approach where the liquid phase is dispensed out of a nozzle to fabricate a meso or micro-scale spatial actuator body layer by layer. This method enables the combinations of a broad range of materials and feasibility of multi-material printing while it is limited in the resolution and modification needed for two-stage materials curing, such as silicone. The DIW method was used to develop a moisture-cured silicone elastomer SPA with minimal voids, high strength, and elongation at break (Plott and Shih 2017). The process parameters were optimized to inhibit void formation during 3D/4D printing of the thinwall silicone SPA. The developed SPA showed a reasonable fatigue life of over 30,000 cycles before failure (Plott and Shih 2017). A robotic soft actuator was 3D/4D-printed with programmable bioinspired architectures using photocurable resins by multi-material DIW (Schaffner et al. 2018). Tunable mechanical properties were achieved by adding vinylterminated silicones of different molecular weights to various silicone-based elastomers, namely Ecoflex 00-30, Dragonskin 30A, and Sylgard 184 (Schaffner et al. 2018). The capability of the DIW method in the combination of multi-material in this work led to the 
development of the viscoelastic silicone inks to form variable stiffness elastomers to improve the control mechanism.

The FDM printers heat thermoplastic filaments to the melting point and then extrude them layer by layer to create 3D-SPAs. The FDM could accommodate a larger group of thermosetting elastomers while having the capability to simultaneously print different and multiple materials. The FDM is used as an effective additive manufacturing method in comparison with SLA, DLP, or SLS, mainly due to its inexpensive and repeatable process as well as much less postprocessing requirements. A modified FDM printer was used to extrude EcoFlex 00-30 (Smooth-On) for developing the SPAs (Morrow, Hemleben, and Menguc 2016). The printed actuator was compared with the mold manufactured method. Though the initial feasibility of the method was confirmed, it was reported that the printed actuators significantly deviated from the desired features, particularly in the wall thickness, leading to lower bending deflection and the force output. A pneumatic finger-like SPA was developed in one step using the FDM method and Filaflex (Recreus) filament (Anver, Mutlu, and Alici 2017). The authors reported an average wall thickness of $0.64 \mathrm{~mm}$. The low-cost SPA was 3D/4D-printed using the FDM technique and commercially available thermoplastic poly(urethane) (TPU), NinjaFlex (NinjaTek, PA) filament (Rosalia, Ang, and Yeow 2018; Yap, Ng, and Yeow 2016). The complex inner features without any supporting materials were utilized to devise the actuator with high force tip performance up to $80 \mathrm{~N}$ (Yap, $\mathrm{Ng}$, and Yeow 2016). A minimum wall thickness of $1.2 \mathrm{~mm}$ reported for this actuator to operate fully airtight. The cyclic fatigue testing on the actuator demonstrated higher durability performance than the 3D-printed SPA developed by projection SLA (Peele et al. 2015). In another study, the Ninjaflex (NinjaTek, PA), with a shore hardness of 85A was used for developing 3D/4D-printed bellow-type SPA where the effects of number and size of the bellows on the curvature bending under the same input pressure were investigated (Keong and Hua 2018). Recently, a bioinspired fin-ray structure for increasing the conformal grasping of the 3D/4D-printed SPA was developed by using commercially available TPU NinjaFlex (NinjaTek, PA) (Tawk, Gao, et al. 2019). The 4D-printed soft gripper showed the enhanced capability of lifting objects with various shapes, stiffness, and textures. A recent modified 3D printing technology, named fused pellets printing (FPP), was developed to make a multi-material SPA with different components with various properties (Khondoker and Sameoto 2019). The SPA was made of optically transparent thermoplastic elastomer pellets of copolymer Kraton G1657 (styrene ethylene butylene styrene (SEBS), Kraton Corporation, USA) with $600 \%$ stretchability and rigid pellets of MM3520 (SMP Technologies Inc., Japan).

A combination of DIW and FDM methods was also devised to additively manufacture a composite SPA from silicone and thermoplastic elastomer (TPE) with potential applications in patient-specific and assistive biomedical devices ( $\mathrm{Ng}$ et al. 2020), such as stent implant and less invasive surgery (Byrne et al. 2018). To achieve this a modified machine with a four-axis, combined FDM and paste extrusion head is required, which would be cumbersome to operate compared to the conventional extrusion-based DIW printers (Byrne et al. 2018).

The SLA is a resin-based 3D printing approach that is using photochemical processes to develop the SPAs with higher resolution and smoothness than the FDM method. However, it is limited to the smaller material library and is quite slow. One of the early fully 3D-printed soft pneumatic actuators was made by a digital mask projection SLA using elastomeric precursor (Spot-E resin, Spot-A Materials, Inc.) and with a thin layer of Sylgard 184 (PDMS) 
adhered to the bottom surface as a polymerization inhibitor (Peele et al. 2015). The life-cycle of the elastomer was found to be $9 \pm 3$ cycles without failure under the performed fatigue test while it has shown a relatively low ultimate strain 1.40 . A high resolution, $\sim 50 \mu \mathrm{m}$, siliconebased elastomeric polydimethylsiloxane (PDMS) pneumatic channel was also fabricated using an SLA printer (Wallin et al. 2017). The actuator was featured with autonomic self-healing in sunlight. The same research team later developed a 4D-printed hydrogel-based poly-Nisopropylacrylamide (PNIPAm) and polyacrylamide (PAAm) SPA using multi-material SLA printing with the capability of perspiration at a higher temperature for self-regulating the desired bending (Mishra et al. 2020).

The SLS uses a laser as the power source to sinter and bind powdered plastic materials, such as polyamide, to create SPAs. Although the 3D/4D-printed SPAs made by SLS are benefiting from high strength and chemical resistance, this method is not suitable for developing the compact SPAs with complex geometries. A case study was performed on fabricating a single body air pressure actuator using the SLS for soft robotic hand application (Scharff et al. 2017). The actuator was 3D-printed using a flexible polyurethane like material to achieve the minimum wall thickness $1 \mathrm{~mm}$. Removing the powder and purging the chamber at the end of printing were mentioned as the main challenges of using this method, impeding the design of various shapes, particularly at the corners of the chamber. Also, a SPA was 3D/4D-printed by using a powder mixture of glass fiber reinforced Nylon, stacking three soft bellow-type actuators using SLS 3D printing technology (Chen et al. 2019).

The PolyJet printing is a fast technique, which sprays the materials using different jetting heads to fabricate high-resolution SPAs. Complex geometries with thin walls could be achieved via this approach though suffering from poor mechanical properties compared with the FDM or SLS and more expensive. The PolyJet printing method was used for 4D printing of a multimaterial finger-like SPA using Object350 Connex3 (Stratasys, Co., Ltd.) and rubber-like TangleBlack material for inflation chambers (Zhu et al. 2018). The wall thickness of the actuator was reported $0.03 \mathrm{~mm}$ while metamaterial design and variable stiffness designs, and glossy finish were featured in the work. In another similar study, a dual-material approach was utilized to develop a 3D/4D-printed SPA using the PolyJet method. The study compared the advantage of dual-material 4D printing of SPA with a single machine. It was revealed that the adverse balling effect of the soft body was reduced significantly in the dual-material printing. Also, a differential pressure three-chambered SPA was made by PolyJet method to increase the workspace of the actuator to a planar motion than a mere bending (Drotman et al. 2018). From a different perspective, a bellow-type pre-stressed SPA was 3D-printed, which functioned analogously to a vacuum SPA (Wang, Torigoe, and Hirai 2017). The actuator was fabricated by the Objet260 Connex3 printer (Stratasys, Co., Ltd.) and a combination of a rubber-like material, TangoPlus, and a hard material, VeroBlue. The novelty of this work was to develop a curled 3D-printed SPA that helps with more maneuverability for grasping tasks in fruit picking. The same group also developed a food handling soft gripper with the same printer and using a mixture of soft TangoBlack+ and hard polymeric, VeroWhite, materials (Wang, Chathuranga, and Hirai 2016). The pre-stressed layer was included in the design to create a larger initial opening space for grasping the foods in various sizes and shapes for packaging purposes. A PolyJet 3D printer (J750, Stratasys Ltd.) was also used to 3D/4D print a tunable stiffness SPA using Agilus30 (Wang, Zhu, et al. 2017). This study investigated the incorporation of VeroClear (Stratasys Ltd.) as shape memory polymer (SMP) to enhance the 
functionality of the SPAs actuators to grasp arbitrary shapes objects in a broad range from 10 g to $1.5 \mathrm{~kg}$ (Zhang, Zhang, et al. 2019).

The DLP is another resin-based 3D printing method, like SLA, which uses light sources to fabricate SPAs. However, its difference with SLA is that the layers are all printed at the same time. The advantage of DLP systems in 3D/4D-printing of SPAs lies in constructing highresolution features that could be directly printed and demand less post-processing steps. On the other hand, there are shortcomings in using DLP due to the limited number of commercial photo-curing silicone elastomers and small print volumes. A SPA was fabricated via a desktop DLP 3D printer and a mixture of TangoPlus FLX 930 and Rhodamine B resins (Ge et al. 2018). The printing process was reported to be completed in less than 30 minutes featuring a microactuator with a $0.2 \mathrm{~mm}$ wall thickness and $87.5 \mu \mathrm{m}$ length. Miniature 3D/4D-printed SPAs were fabricated using the DLP-based multi-material printing technology for soft robotics. The manufacturing challenges caused by the void formation in millimeter scale were reported to be rectified through the appropriate selection of photo absorber, its concentration, layer thickness, exposure time to UV light, and their effects on the mechanical properties of the SPA (Zhang, Ng, et al. 2019). A highly stretchable ultraviolet curable resin was formulated by mixing epoxy aliphatic acrylate (EAA), and an aliphatic urethane diacrylate (AUD) and was printed using a DLP process for developing 4D-printed pneumatically actuated grippers (Patel et al. 2017). In a similar study, the stretchability and stiffness of a commercially available UV curable elastomer, TangoPlus, was modified, to withstand more than 1000 times of loading/unloading cycles (Hingorani et al. 2019). This was achieved by mixing two chemical additives, EAA as mono-acrylate based linear chain builder and AUD crosslinker. The outstanding stretchability of the introduced elastomer led to much larger bending deformation in the 3D/4D-printed soft actuator compared to other similar works. A photo resin made from bis(propylacrylamide)-poly(dimethylsiloxane) (PDMS-DMAA) was also used for DLP printing of soft pneumatic actuator with high elongations of up to $472 \%$ under tensile load (Thrasher, Schwartz, and Boydston 2017).

In general as highlighted in Table 1, the DIW has shown promising results for 3D/4D printing of SPAs with minimal voids, controlled variable stiffness, acceptable fatigue properties, and high strength and elongation at break compared to the DLP, SLS, SLA, and PolyJet techniques. The FDM approach showed higher fatigue durability performance than the 3D-printed SPA developed by projection SLA (Peele et al. 2015). SLA is not the best method in terms of achieving the minimum wall thickness, which hinders its capability for achieving higher bending deflection and blocking force. However, the SLA method has shown significant performance in the fabrication of high-resolution 3D/4D-printed SPAs despite its weakness in durability and limited life-cycle. This method, also, is suggested to be suitable for the actuators with self-healing properties. The SPAs made by the SLS method have shown higher strength compared to other 3D printing techniques but were not promising in the design of various complex geometries due to the purging difficulty of the chamber at the end of printing. Besides, the materials used in SLS are not elastomers but thermoplastics that could yield and plastically deform under bending. The PolyJet method has featured capability of multi-material, functionally graded, and glossy surface finish printing at a higher cost than DIW and FDM while suffering from poor mechanical properties compared with the FDM or SLS. The DLP is confirmed as the method of choice for miniaturized highly stretchable 3D/4D-printed micro SPAs, however, is not suitable for large volume and longer life-cycle SPA applications. 
Table 1. 3D printers and materials used for 3D/4D printing bending-type SPAs.

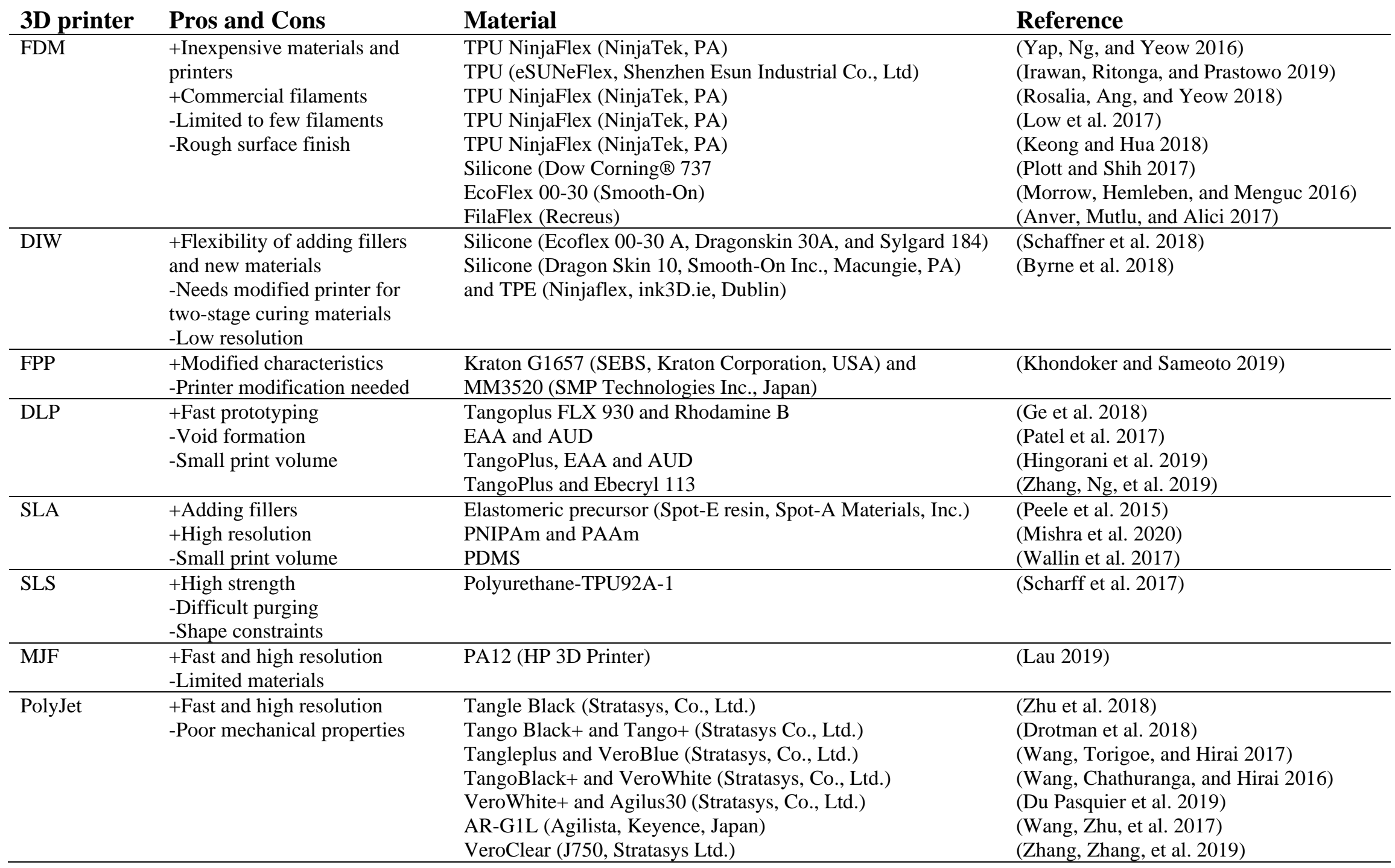




\section{Modeling of 3D/4D-printed bending-type soft pneumatic actuators}

The two main types of bending-type SPAs are identified in the literature. The first group is made by one, or even grouping of two or three of antagonistic fiber-reinforced chambers that control the bending of the actuators through pressure difference between the chambers also called tubular fiber-reinforced actuators (Connolly, Walsh, and Bertoldi 2017; Zhou, Chen, and Wang 2017). However, the second group is designed based on pressurizing air chambers formed by an asymmetric cross-section, with the inextensible bottom layer and an extensible top layer, which converts the pressure change into bending toward the side with the higher bending stiffness, known as bellow-type SPAs (Tawk, Gao, et al. 2019). The pneumatic chambers introduced in these SPAs can protect the walls from excessive deformation and thus induce the development of reliability against failure in higher input pressure. The bellow-type SPAs have been mainly focused in 4D printing due to several reasons (Drotman et al. 2018; Yap, $\mathrm{Ng}$, and Yeow 2016); for creating rapid motions by unfolding the bellows, undergoing less radial expansion, which in turn minimizes failure and improves the durability, achieving full bending at lower pressures, and higher speed.

The FEA approaches have been broadly used for simulating and modeling the SPA's kinematics and dynamics, but, the computational burden associated with these methods to achieve accurate results is always a matter of disputation that does not deter the researchers from exploring the analytical models first. Hence, in this study too, the analytical modeling and underlying mechanics of 3D/4D-printed bending-type SPAs are initially presented.

\subsection{Analytical kinematic model}

There are have been several analytical modelings of 3D/4D-printed SPAs developed in the literature. A principal strain field method was developed to realize the target deformation of SPAs (Ding et al. 2019). The heuristic approach utilized in the study could be more beneficial in 3D/4D-printed SPAs where material distribution was incorporated into the optimization of the constraints of the pneumatic chambers to achieve the target deformation. An analytical model of a three-chambered bellow-type actuator, including the forward and inverse kinematics, was also developed to analyze the extended motion of such actuators in 3D space rather than the planar model (Drotman et al. 2018). A rapid kinematic modeling of microtube SPA was also developed based on the line-segment model and the multi-segment EulerBernoulli's beam model with the claimed less computational burden than the FEA (Ji et al. 2019).

Among all the analytical models developed for relating the input pressure to the bending deflection of the SPAs, a straight forward model based on Euler-Bernoulli is presented here. Assuming the SPA has a uniform deformation under pressure input, the bending model shown in Fig. 4(a) could be developed (Zhong, Hou, and Dou 2019). Thus, the geometric relationship could be defined as follows:

$$
\alpha=\frac{\beta}{2}, \beta=\frac{L}{R}, L=L_{0}+\Delta L
$$

where $\alpha$ is the bending angle under pressure input, $\beta$ is the central angle of the actuator arc, $R$ is the bending radius, $\mathrm{L}$ is the axial length of the upper finger after deformation, $\Delta \mathrm{L}$ is the growth of the upper side, and $\mathrm{M}_{\mathrm{i}}$ is the bending moment generated by input air. Referring to the Euler-Bernoulli law: 
$\frac{M_{i}}{E I}=\frac{1}{R}$

where $\mathrm{E}$ and I are the modulus of elasticity and the second moment of area of the actuator's cross-section, respectively. The corresponding force of the input pressure $(P)$ that acts on the internal surfaces of the chamber is:

$F_{i}=P A_{i}$

where $A_{i}$ is the cross-sectional area of the actuator. The axial elongation, $\Delta L$, of the top surface generated by $F_{i}$ can be obtained as:

$$
\Delta L=\frac{F_{i} L_{S}}{E A_{c}}=\frac{P A_{i} L_{S}}{E A_{c}}
$$

where $L_{s}$ is the length of the chamber and the distance between adjacent chambers. The moment of the entire SPA produced by input pressure, acts into two different cross-sectional areas, as shown in Fig. 4(b). $O$ is the location of the cavity centroid, $N$ is the neutral axis location of the cross-section, and $h_{i}$ is the effective distance between points $O$ and $N$. The internal compressed air acts on the point $O$ generates a bending moment $M_{i}$ to bend the actuator toward the bottom part at point $N$. Thus, by combining formulas (1)-(4), the relationship between the bending angle $\alpha$ and the input pressure value $P$ could be calculated as:

$$
\begin{aligned}
\alpha & =\frac{L_{0}+\Delta L}{2 R}=\frac{A_{i}^{2} h_{i} L_{s}}{2 E^{2} I A_{c}} P^{2}+\frac{A_{i} h_{i} L_{0}}{2 E I} P \\
& =m P^{2}+n P
\end{aligned}
$$

where $m$ and $n$ could be attained via curve fitting from experimental data.
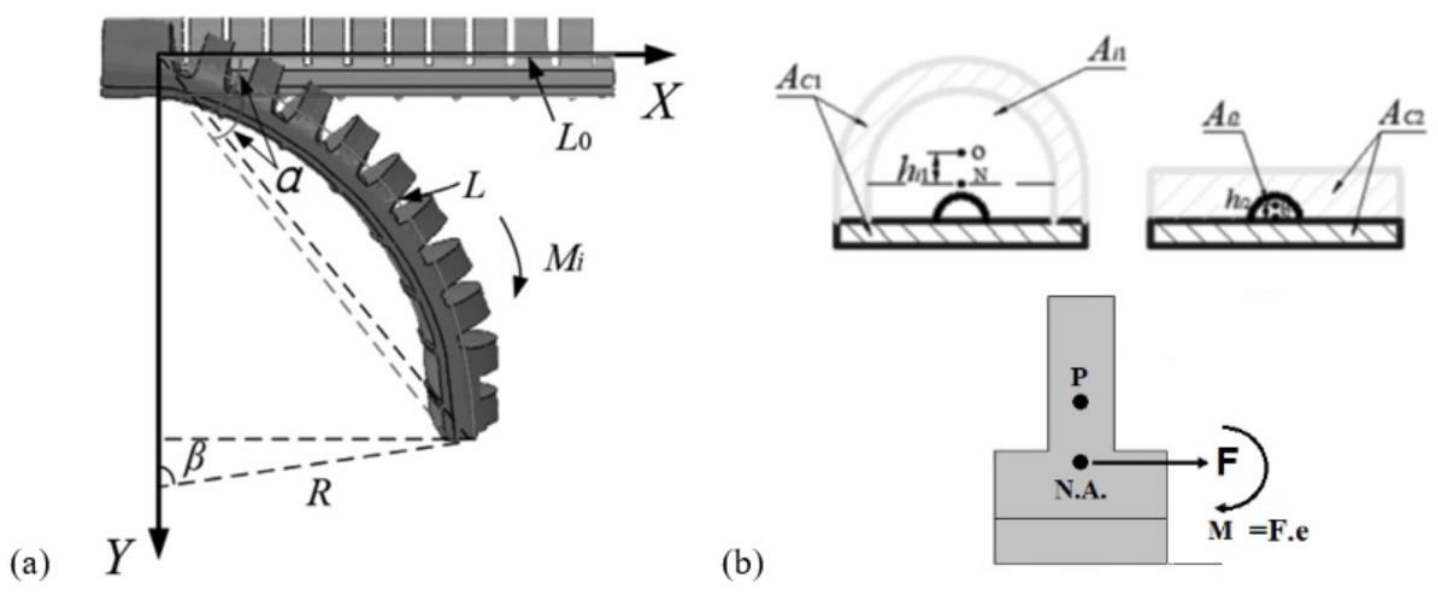

Fig. 4. (a) Bending angle of a 3D/4D-printed SPA (b) Cross-section view of the single and adjacent chambers. Reproduced from (Zhong, Hou, and Dou 2019) with the permission of Elsevier.

Kinematic modeling of SPA resembles a flexible manipulator with many degrees of freedom (DOF) and continuous deformation. Therefore, a simplified method based on the discrete rigid elements and transformation matrices were applied with acceptable accuracy (Zolfagharian et al. 2018; Zhong, Hou, and Dou 2019). Having calculated the bending angle of the actuator 
corresponding to the input air pressure, the kinematic model of the actuator could be derived to find the deformation of the whole body. A simplified model could divide the actuator into $q$ equal rigid links assuming a uniform pressure along with the pneumatic chambers and constant distances in the circular arc direction between the adjacent joints surface during deformation. Since the curvature mainly occurs in the gap between neighboring chambers, the joints surfaces are assumed at the midpoint between the adjacent chambers (Zhong, Hou, and Dou 2019).

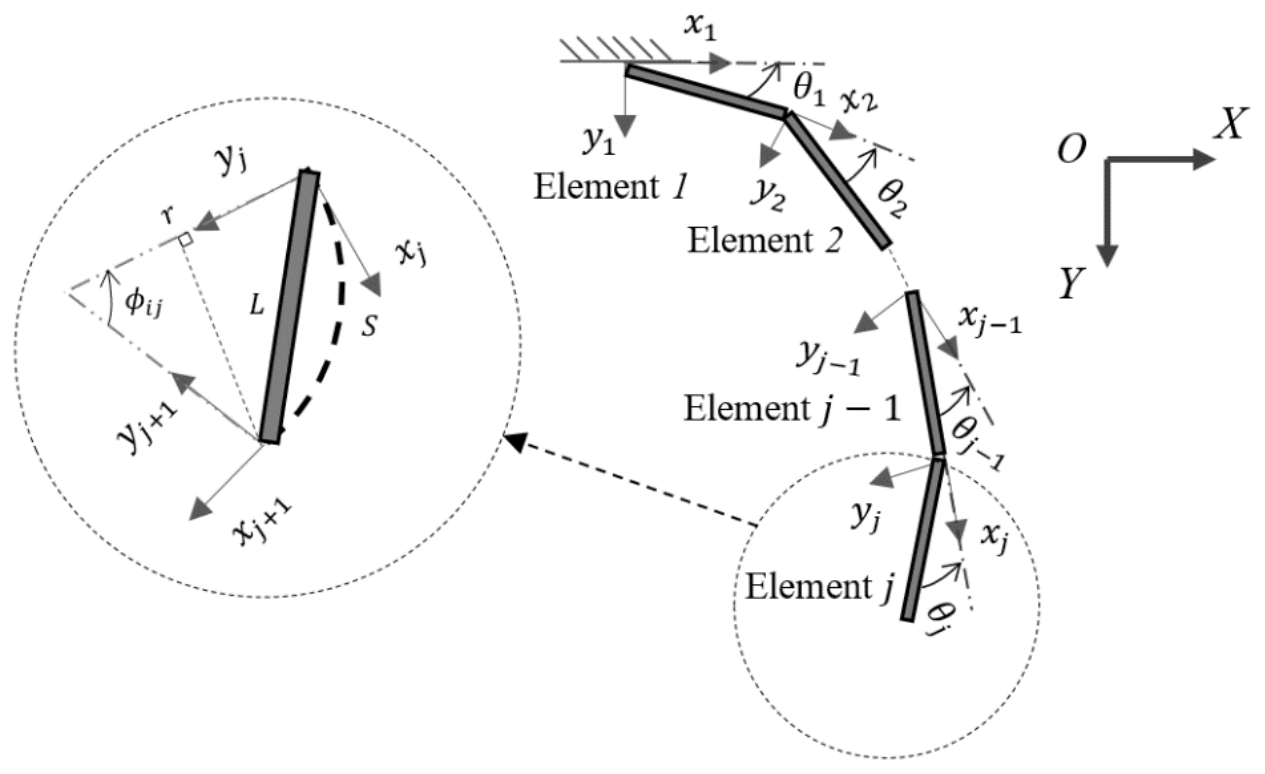

Fig. 5. Discrete rigid elements kinematic modeling of a 3D/4D-printed SPA.

The pneumatic actuator divided into $q$ equal length 3D-printed soft chambers is shown in Fig. 5. From the simplified geometry shown in Fig. 5 the relationship between the arc length, $S$, and the rigid link length of $i^{\text {th }}$ chamber $L_{i}$, is derived as:

$\varphi_{i j}=\theta_{i}, L_{i}=\frac{2 \sin \left(\frac{\varphi_{i j}}{2}\right)}{\varphi_{i j}}$

Using the transformation matrices:

${ }^{1} T_{j}={ }^{0} T_{1} \cdot{ }^{1} T_{2} \ldots{ }^{j-1} T_{j}$

where

${ }^{j-1} T_{j}=\left[\begin{array}{cc}{\left[R_{\theta_{z}}\right]_{(3 \times 3)}} & {[P]_{(3 \times 1)}} \\ {[0]_{(3 \times 1)}} & 1\end{array}\right]=\left[\begin{array}{cccc}\cos \theta_{j} & -\sin \theta_{j} & 0 & m_{j} \\ \sin \theta_{j} & \cos \theta_{j} & 0 & n_{j} \\ 0 & 0 & 1 & 0 \\ 0 & 0 & 0 & 1\end{array}\right], j=1, \ldots, q$

$m_{j}$ and $n_{j}$ are calculated as:

$m_{j}=\frac{s\left(\sin \theta_{j}\right)}{\theta_{j}}, n_{j}=\frac{s\left(1-\cos \theta_{j}\right)}{\theta_{j}}, j=1, \ldots, q-1$.

Therefore, Eq. (8) could be rewritten as:

${ }^{j-1} T_{j}=\left[\begin{array}{llll}R_{j} & P_{j} & 0 & 1\end{array}\right]^{T}$ 
where

$$
\begin{aligned}
R_{j} & =m_{1}+\sum_{k=2}^{j-1}\left[m_{k} \cos \left(\theta_{1}+\theta_{2}+\cdots+\theta_{k}\right)-n_{k} \sin \left(\theta_{1}+\theta_{2}+\cdots+\theta_{k}\right)\right] \\
P_{j} & =n_{1}+\sum_{k=2}^{j-1}\left[m_{k} \sin \left(\theta_{1}+\theta_{2}+\cdots+\theta_{k}\right)+n_{k} \cos \left(\theta_{1}+\theta_{2}+\cdots+\theta_{k}\right)\right] \\
j & =3, \ldots, q .
\end{aligned}
$$

\subsection{Geometrical parameters effects on the bending angle of soft pneumatic actuators}

Bellow geometry is a key factor in characterizing the deflection of the SPAs that could be a luxury of 3D/4D-printed SPAs too. The common geometries of bellows and their analytical stiffness model for hard materials are broadly investigated in the past (highlighted in Fig. 6). However, more understanding of the relation between the SPA deflection and the bellow shape was revealed in recent studies (Lau 2019; Stano, Arleo, and Percoco 2020). Using both FEA and the experimental results, it was found that the U-shaped bellow outperformed the other geometries by far in terms of achieving the maximum deflection for the same input pressure (Lau 2019). The comparison results of the other bellow geometries were reported as:

$$
\delta_{U \text {-shaped }}>\delta_{\text {Elliptical }}>\delta_{\text {Rectangular }}>\delta_{\text {Crimped-plates }}>\delta_{S \text {-shaped }}>\delta_{\text {Triangular }}
$$

where $\delta$ is the deflection of the SPA under the same input pressure.

In another study, different shapes of chambers were 3D printed and their effects on the bending angle of the SPAs were investigated (Stano, Arleo, and Percoco 2020). Experimental results revealed that the best bending performance was achieved by the R-type (highlighted in Fig. 7) design compared to others.

$$
\delta_{R}>\delta_{S}>\delta_{D} \cong \delta_{B}
$$

(Stano, Arleo, and Percoco 2020)

The R- and S-type, respectively, exhibited the largest bending angles with little difference at the pressure range of 0-3 bar. However, the B- and D-type demonstrated smaller bending angles than R- and S-type. At pressures higher than 4 bar, however, the D-type out-raced the B-type. Generally, the authors related these different bending angles of the actuators to the alignment of the chamber walls with the airflow direction. 

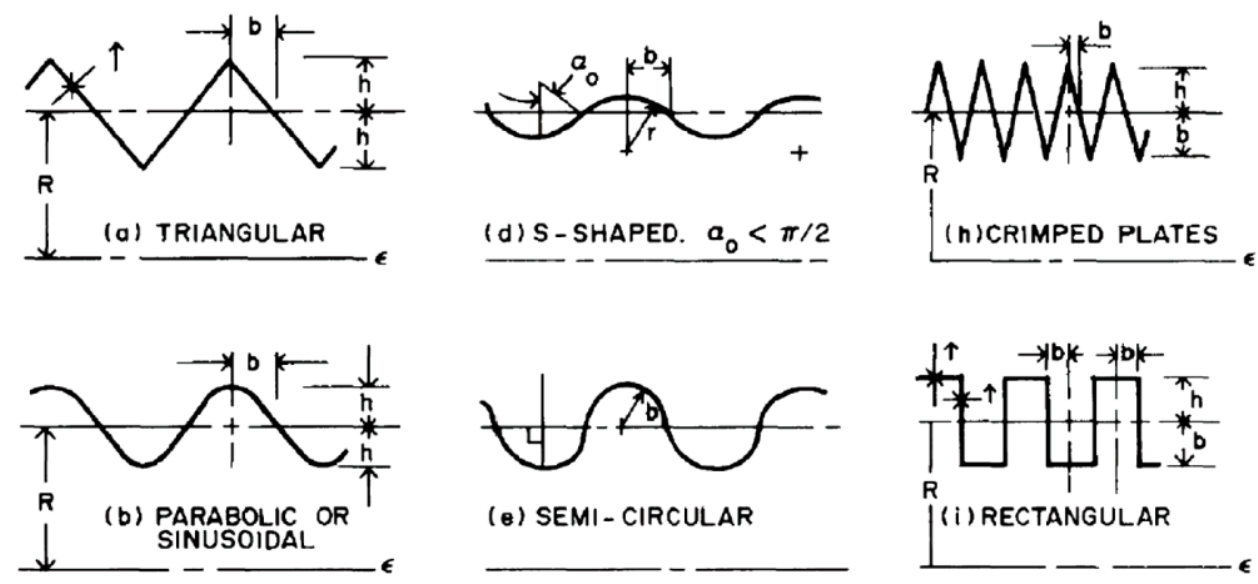

(e) SEMI- CIRCULAR
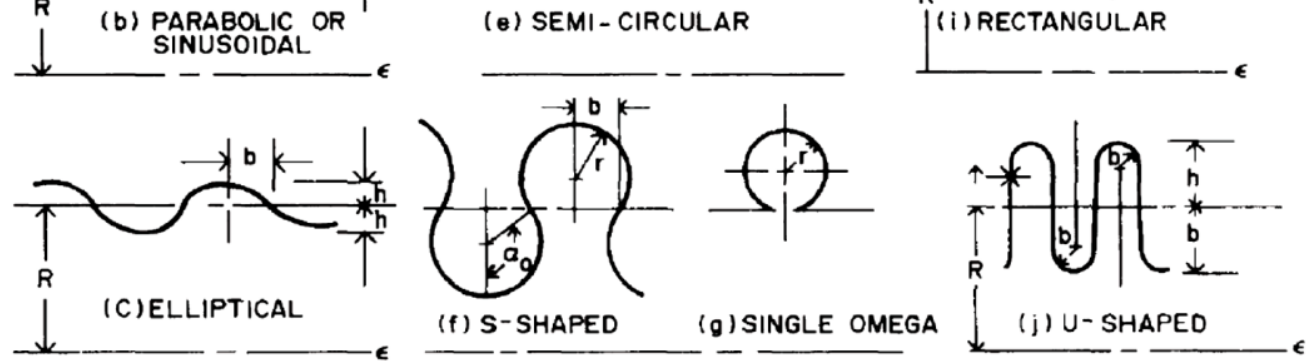

Fig. 6. Various geometries for linear bellow-type actuators, reproduced from (Wilson 1984) with permission of Elsevier.
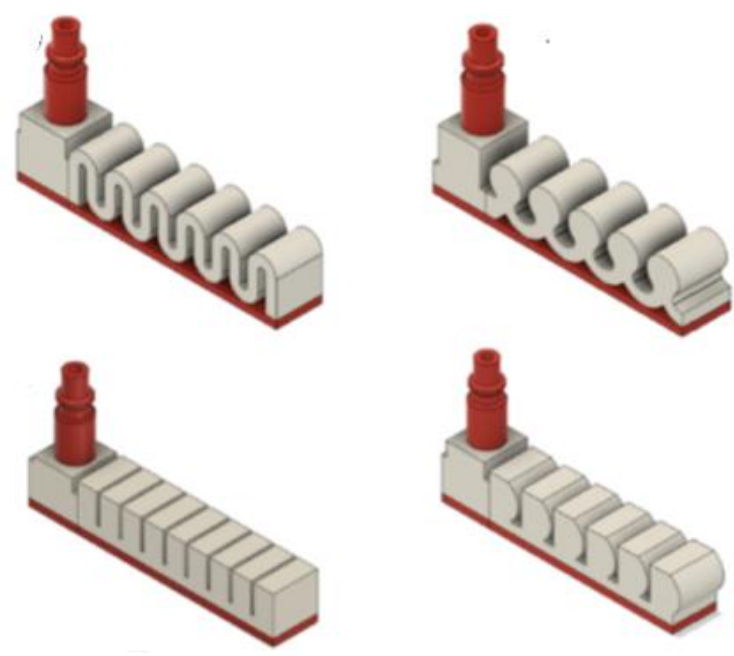

Fig. 7. Different designs of 3D/4D-printed SPAs: (a) S-type, (b) B-type, (c) R-type, and (d) Dtype, reproduced from (Stano, Arleo, and Percoco 2020) with permission of MDPI under the terms of the Creative Commons CC BY license.

Besides, the variation of SPAs deflection with the cross-section geometries was investigated (Hu et al. 2018). The comparison of results of the cross-section geometries was reported as:

$$
\delta_{\text {Honeycomb }} \cong \delta_{\text {Rectangular }} \cong \delta_{\text {Semi-circlular }}>\delta_{\text {Circular }}
$$

(Hu et al. 2018)

where $\delta$ is the deflection of the SPA under the same input pressure. It was found that while the honeycomb, rectangular, and semi-circle cross-sections have led to the more or less the same 
extent of deflection, the actuator with circular cross-section has been the last effective one in this regard.

The deflection of 3D/4D-printed SPAs is highly dependent on the design parameters of the chambers, including bellow gap, width, and wall thickness, and bottom layer thickness (Auysakul et al. 2020; Demir et al. ; Sun et al. 2019). These relations have been studied earlier and the effects of these parameters are shown in Fig. 8 (Hu et al. 2018). It was observed that the deflection of the SPA has a parabolic correlation with bottom layer thickness. Also, it was shown that the deflection of the SPAs decreases with increasing the gap size and the wall thickness of chambers in the bellow. The wall thickness is a crucial variable in the design of 3D/4D-printed SPAs. A thin wall thickness leads to a lower life-cycle while a thick wall leads to a smaller bending angle under the same input pressure. Therefore, there should be a balanced design for an optimized value of the wall and bottom layer thickness to avoid the rupture while achieving the full bending of the SPAs. Therefore, simultaneous consideration of wall and bottom layer thickness is recommended at the design stage (Hu et al. 2018).

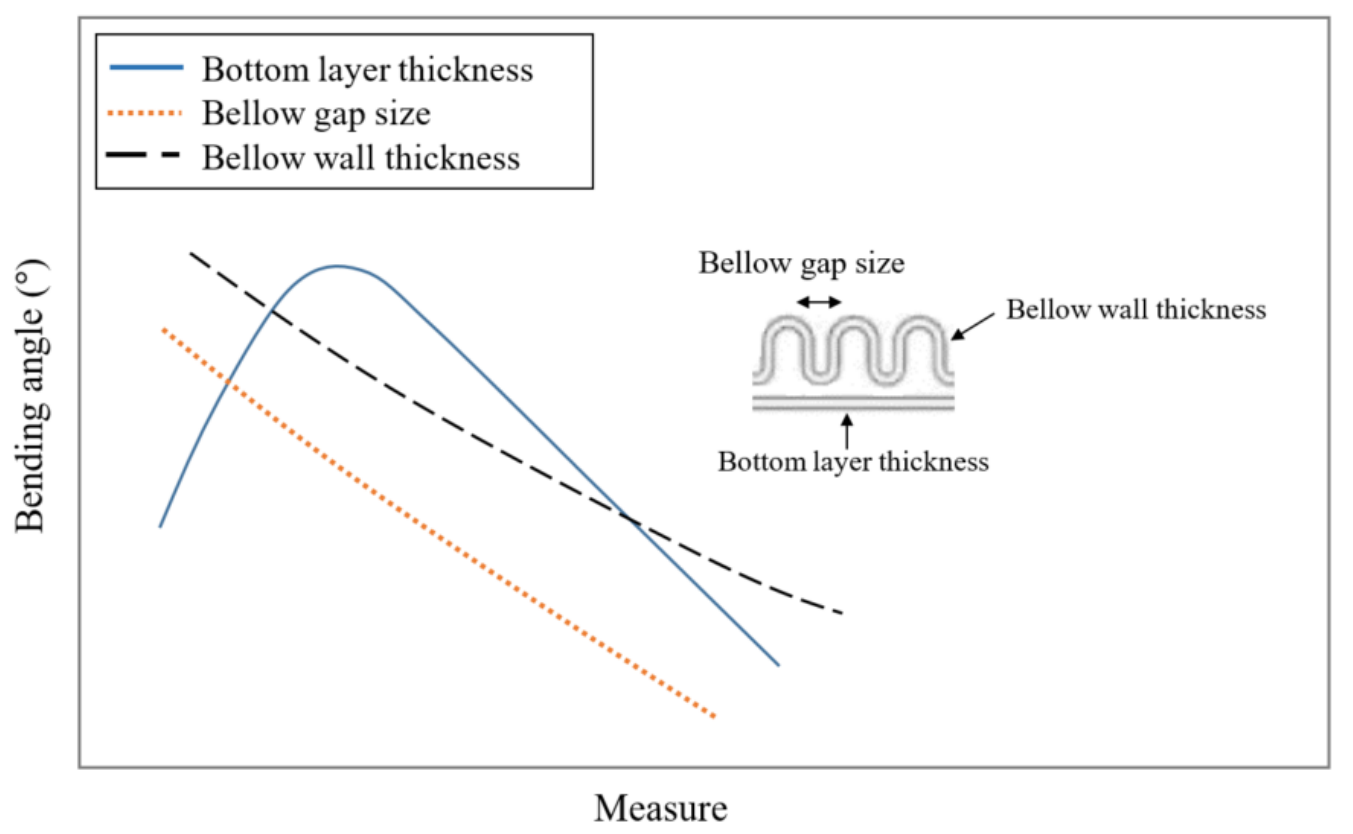

Fig. 8. The effects of different geometrical parameters on the bending angle of SPAs.

The effect of asymmetry on the bending of 3D/4D-printed SPAs was investigated (Dilibal, Sahin, and Celik 2018). It was found that the tapered design with gradually reduced height toward the tip, which bio-mimics the elephant trunk led to a more bending angle compared to non-tapered. It was also found that the height of the chamber could be optimized for the amount of material used to produce the same extent of deflection and tip force in the bellow type 3D/4D-printed SPA (Auysakul et al. 2020). The bending effects of SPAs under various crosssection ratios were investigated via non-linear FE simulations. The cross-section ratio was defined between the size of the first and last chamber width. It was reported that increasing the cross-section ratio increases the bending angle, conversely, a decrease in cross-section ratio results in significant bending angle reduction. The authors discussed that the cross-section ratio of SPAs could be optimized over the same length depending on the applications to obtain 
optimal curvature for grasping different objects. A genetic algorithm (GA) optimization method was also used to find the optimum design of the actuator for the target task of grasping (Sun et al. 2019). A blend of analytical and FE modeling was proposed to design a SPA that matches the target trajectory by adjusting the design parameters (Sun et al. 2019).

\subsection{Analytical blocking/tip force model}

The effect of design parameters on the blocking/tip-force of 3D/4D-printed bending-type SPAs was investigated. The outcomes of the studies revealed that the decrease in both bellow wall thickness and sidewall thickness, as well as the increase in cross-sectional width, resulted in increased tip-force at the same input pressure (Yap, $\mathrm{Ng}$, and Yeow 2016). The effect of the length of the 3D/4D-printed SPA on the blocking force at the tip was also proven to be directly proportional (Anver, Mutlu, and Alici 2017).

Besides, the effect of different design parameters, including width, length, bottom layer thickness, bellow wall thickness, number of chambers, and cross-section geometry (Anver, Mutlu, and Alici 2017) on the tip force of 3D/4D-printed SPAs was investigated (highlighted in Fig. 9). It was found that the tip force of the actuator increased with the increase of the width, length, and the number of chambers. However, the increase of the bottom layer thickness and bellow wall thickness reduce the tip force of the SPAs. Also, the variation of SPAs tip force with the cross-section geometries was investigated (Hu et al. 2018). The comparison results of the cross-section geometries were reported as follows:

$$
F_{\text {Triangle }}>F_{\text {Honeycom }}>F_{\text {Semi-circlular }}
$$

where $F$ is the tip force of the SPA under the same input pressure.

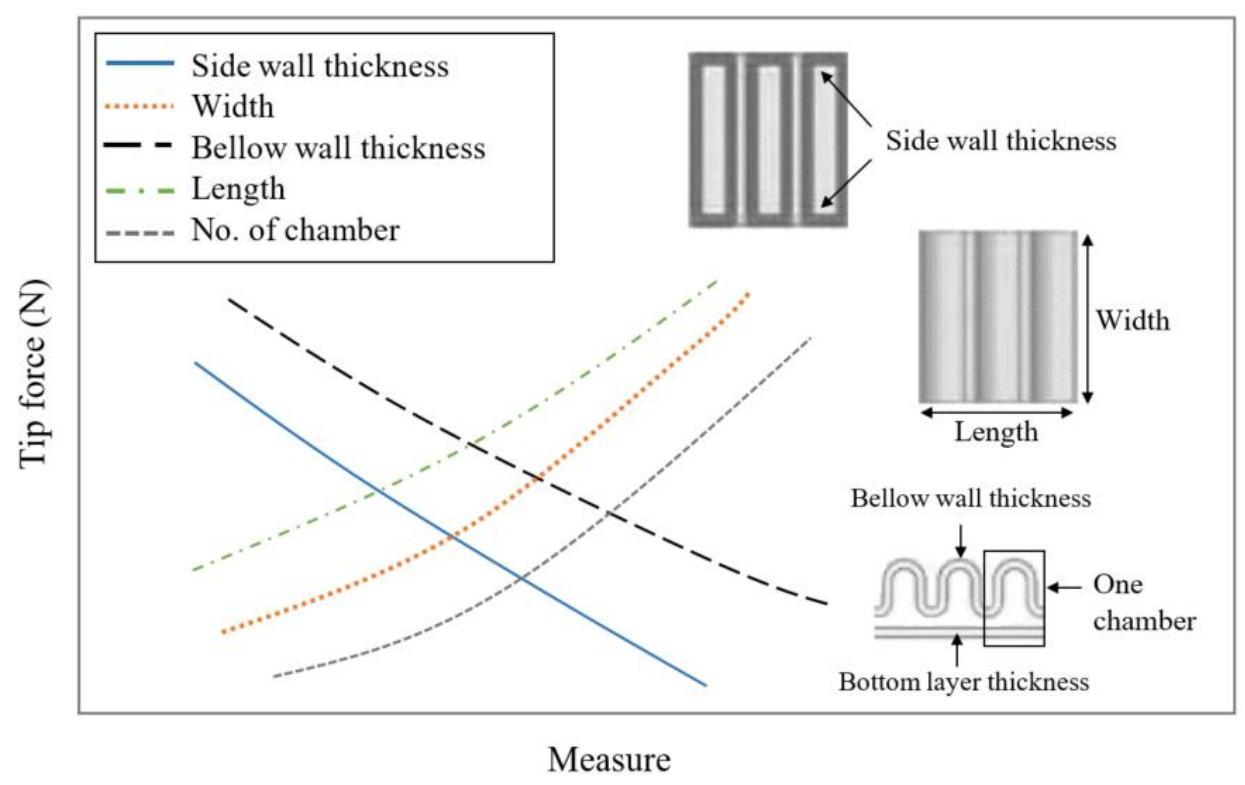

Fig. 9. The effects of different geometrical parameters on the tip force of SPAs.

Also, it was reported that using the experimental measurement of the tip force the effective modulus of elasticity $(E I)$ of the SPA could be found to later estimate the theoretical blocking/tip force of the SPA at different bending angles (Alici et al. 2018). Using (2) and (3), and knowing the flexural rigidity $(E I)$ of the actuator at the given bending angle we have: 
$\frac{M_{B}}{E I}=\frac{1}{R} \cong \frac{\theta}{L}$

where $M_{B}$ is the bending moment at the tip, $R$ is the radius of curvature, $L$ is the length of the actuator, and $\theta$ is the bending angle. Knowing $M_{B}=F_{B}$. $L$, the blocking force or tip force of 3D/4D-printed SPAs could be theoretically written as:

$$
F_{B}=\theta \frac{E I}{L^{2}}
$$

However, it is often impractical to find an accurate value of flexural rigidity for such systems, particularly for multi-material actuators (Alici et al. 2018). Therefore, usually, the experimental data is collected initially for flexural rigidity by rearranging (13) as:

$$
E I=L^{2} \frac{F_{B}}{\theta}
$$

Then the flexural rigidity will be used for tip force estimation.

\section{Finite Element Analysis of 3D/4D-Printed Bending-type Soft Pneumatic Actuators}

Hyperelastic materials with high flexibility and capability of withstanding large strains, such as silicone and thermoplastic elastomers, are the most common materials used in the fabrication of 3D/4D-printed SPAs (Moseley et al. 2016). These materials demonstrate rubber elasticity with quite large and reversible nonlinear deformation, several times of their initial length, under a relatively low force. Their mechanical behavior cannot be characterized by a constant elastic modulus (highlighted in Fig. 10). They possess incompressible characteristics, which makes them retain their overall volume under applied stress (Hu et al. 2018; Hu 2019). The mechanical behavior of hyperelastic materials is complex therefore warranting constitutive mathematical strain energy function models to be employed to predict their elastic behavior via tensile test experimental data (Rivlin 1948; Ogden 1972; Ponte Castaneda 1989; Gent 1996). To estimate the nonlinear large deformation of these materials using FEA various strain energy models were employed and compared (Drozdov 2007; Kim et al. 2012; Marckmann and Verron 2006). Yet, not a single best model was reported as the selection of the best model is subjective to the application, and the material used in the SPA. Isotopic hyperelasticity models, including, Arruda-Boyce (Arruda and Boyce 1993) and Blatz-Ko, (Blatz and Ko 1962) were developed based on the incompressible hyperelastic material response at the molecular scale, while other models, including Mooney-Rivlin (Rivlin 1948), Ogden (Ogden 1972), Neo-Hookean (Ponte Castaneda 1989), and Yeoh (Yeoh 1993) reflect the rubber-like behavior of these materials, known as extended-tube models. Further details of the comparison of these models are discussed in the following. 


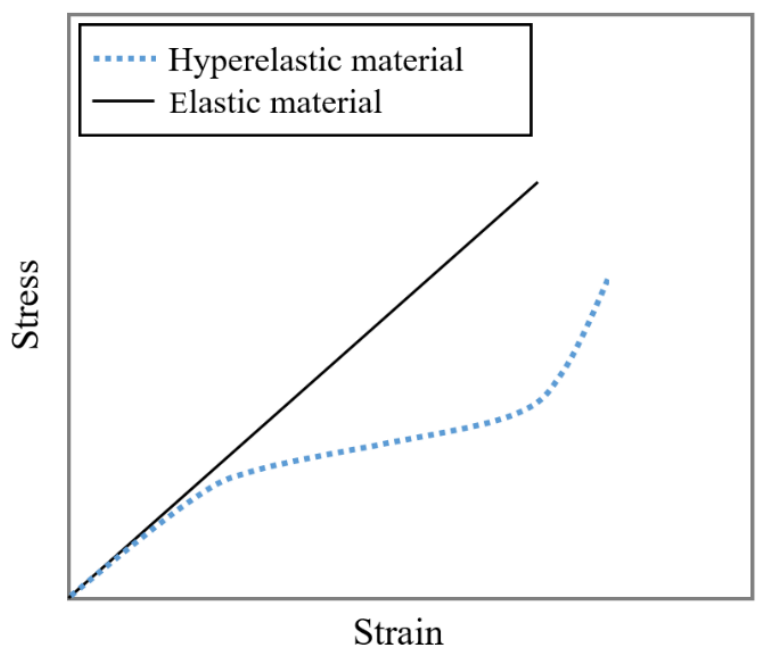

Fig. 10. A schematic diagram of the difference in the stress-strain behavior between hyperelastic and linear elastic materials.

The energy is internally stored over the volume of a material element when is deformed by an external load. This internal energy is related to material strain and is known as the strainenergy. Consider an object under tension, where a volume element of the object is subjected to uniaxial stress, $\sigma$ (Galley, Knopf, and Kashkoush 2019). Assuming there is no energy loss, the stored strain energy, $\Delta U(E)$ in the constant volume element could be calculated based on the external work as:

$$
\Delta U(E)=\frac{1}{2} \sigma \epsilon \Delta V
$$

where $\epsilon$ and $\Delta V$ are the strain and volume change of element, respectively. Then, the strain energy density formula could be derived from (15) as:

$$
W(E)=\frac{\Delta U(E)}{\Delta V}=\frac{1}{2} \sigma \epsilon
$$

For a volume element subjected to three principal stresses, (16) could be rewritten such that each principal stress contributes a portion of the total strain-energy density as:

$$
W(E)=\frac{1}{2} \sigma_{1} \epsilon_{1}+\frac{1}{2} \sigma_{2} \epsilon_{2}+\frac{1}{2} \sigma_{3} \epsilon_{3}
$$

The hyperelastic nonlinear mechanical behavior of the elastomers commonly used in 3D/4Dprinted SPAs are characterized in terms of strain energy density function by assuming the isotropic and incompressible behavior for rubber-like elastomers. Therefore, the strain energy density function, $W(E)$, is dependent on strain invariants of Green deformation tensor, $I_{1}, I_{2}, I_{3}$, and is expressed as follows:

$$
W(E)=f\left(I_{1}, I_{2}, I_{3}\right)
$$

where

$$
\begin{aligned}
& I_{1}=\operatorname{trace}(E)=\lambda_{1}^{2}+\lambda_{2}^{2}+\lambda_{3}^{2} \\
& I_{2}=\frac{1}{2}\left(\operatorname{trace}(E)^{2}+\operatorname{trace}\left(E^{2}\right)\right)=\lambda_{1}^{2} \lambda_{2}^{2}+\lambda_{2}^{2} \lambda_{3}^{2}+\lambda_{1}^{2} \lambda_{3}^{2}
\end{aligned}
$$


$I_{3}=\operatorname{det}(E)=\lambda_{1}^{2} \lambda_{2}^{2} \lambda_{3}^{2}$

and

$\lambda_{i}=1+\epsilon_{i}$

The $\lambda_{1}, \lambda_{2}, \lambda_{3}$ are the principal stretch ratio of Cauchy-Green tensor, and $\epsilon_{i}$ is the uniaxial strain. The strain-energy density function, $W(E)$, in (17) is defined as a sum of two terms (Holzapfel 2010):

$$
W(E)=W_{\text {iso }}\left(\bar{I}_{1}, \bar{I}_{2}\right)+W_{v}(J)
$$

where the isochoric term, $W_{i s o}$, represents the energy needed to deform or distort the element while preserving volume, and the volumetric term, $W_{v}$, represents the energy needed to change in volume of the element with no change in shape. Also, $\bar{I}_{1}=I_{1} / J^{2 / 3}, \bar{I}_{2}=I_{1} / J^{4 / 3}$, and $J=$ $\sqrt{I_{3}}$ is the volume ratio for incompressible materials that is equal to 1 .

The common strain energy formula utilized in FEA of SPAs are listed in Table 2, where $C_{i j}$ and $d_{i}$ are material constants related to shear and bulk moduli, respectively. The Yeoh model is a phenomenological model used for simulating large nonlinear deformation while fitting the mechanical behavior of other deformation modes, such as uniaxial compression and shear, according to the data of uniaxial tensile test (Yeoh 1993). The Yeoh model is founded on the negligible influence of strain tensor invariants $I_{2}$ on the strain energy for nearly incompressible materials. The Mooney-Rivlin model is one of the most popular strain energy density models that could be used for fitting the nonlinear stress-strain curves of a broad range of hyperelastic materials based on the principal strain invariants in two, three, five or nine parameter models (Mooney 1940). The Ogden model is developed and used to fit the non-linear stress-strain behavior of large strain materials like biological tissue based on three principal stretches where $\bar{\lambda}_{i}=J^{-1 / 3} \lambda_{i}$, and $\mu_{i}$ and $\alpha_{i}$ are temperature-dependent parameters of materials. The measurability of the principal stretches in Ogden is deemed its advantage over the MooneyRivlin model. The Neo-Hookean model is a particular case of the Ogden model by limiting the number of terms in strain energy function to 1 and the fitting parameter $\alpha_{i}=2$ (Ponte Castaneda 1989). This model is used to fit into nonlinear materials with small strains where $\mu$ is the initial shear modulus of the material and $d$ is the material incompressibility parameter. The polynomial model is a phenomenological hyperelastic material model suitable for describing the behavior of filled elastomers (Forni, Martelli, and Dusi 1999). The ArrudaBoyce, also known as the eight-chain model, was developed based on the evaluation of a cubic volume element with eight chains and is another suitable model for incompressible elastomeric materials (Arruda and Boyce 1993; Boyce 1996). The Blatz and Ko is a compressible model developed for finite strain modeling of more foam-like rubbery materials (Blatz and Ko 1962). Given the nature of 3D printing, the actuators could show anisotropic properties. Therefore, material properties, like Young's modulus and Poisson's ratio of 3D-printed dumbbell test specimens in different directions are commonly characterized using a uniaxial tensile test for FEA. Expectedly, the specimens printed longitudinally demonstrate the highest stress, while the transverse printed specimens experienced the lowest stress. In several studies, only the longitudinal tests results were used for the constitutive model fitting of hyperelastic materials since it was justified that in the bellow-type pneumatic actuators the majority of the strains 
occurred in the longitudinal direction of the bellows of the actuators during actuation (Yap, $\mathrm{Ng}$, and Yeow 2016). Then the curve fitting methods (highlighted in Fig. 11) available in FE packages, such as ANSYS and ABAQUS, are employed to find the material constants from the stress-strain experimental data to characterize nonlinear behavior of hyperelastic materials in 3D/4D-printed SPAs (highlighted in Figs. 11 and 12).

Table 2. Energy model formula applied for FEA of 3D/4D-printed SPAs.

\begin{tabular}{|l|l|}
\hline Energy Model & Formula \\
\hline $\begin{array}{l}\text { Yeoh } \\
\text { (Yeoh 1993) }\end{array}$ & $\begin{array}{l}W=\sum_{i=1}^{3} C_{i 0}\left(\bar{I}_{1}-3\right)^{i}+\sum_{i=1}^{3} \frac{1}{d_{i}}(J-1)^{2 i} \\
\text { Two-parameter incompressible material model: } \\
W=C_{10}\left(I_{1}-3\right)+C_{20}\left(I_{1}-3\right)^{2}\end{array}$ \\
\hline & $W=\sum_{i, j=0}^{N} C_{i j}\left(\bar{I}_{1}-3\right)^{i}\left(\bar{I}_{2}-3\right)^{j}+\sum_{i=1}^{N} \frac{1}{d_{i}}(J-1)^{2 i}$ \\
& $\begin{array}{l}\text { Two-parameter incompressible material model: } \\
W=C_{10}\left(I_{1}-3\right)+C_{01}\left(I_{2}-3\right) \\
\text { Three-parameter incompressible material model: }\end{array}$ \\
& $W=C_{10}\left(I_{1}-3\right)+C_{01}\left(I_{2}-3\right)+C_{11}\left(I_{1}-3\right)\left(I_{2}-3\right)$ \\
Mooney-Rivlin & Five-parameter incompressible material model: \\
(Mooney 1940) & $W=C_{10}\left(I_{1}-3\right)+C_{01}\left(I_{2}-3\right)+C_{20}\left(I_{1}-3\right)^{2}+$ \\
& $C_{11}\left(I_{1}-3\right)\left(I_{2}-3\right)+C_{02}\left(I_{2}-3\right)^{2}$ \\
Nine-parameter incompressible material model: \\
$W=C_{10}\left(I_{1}-3\right)+C_{01}\left(I_{2}-3\right)+C_{20}\left(I_{1}-3\right)^{2}+$
\end{tabular}



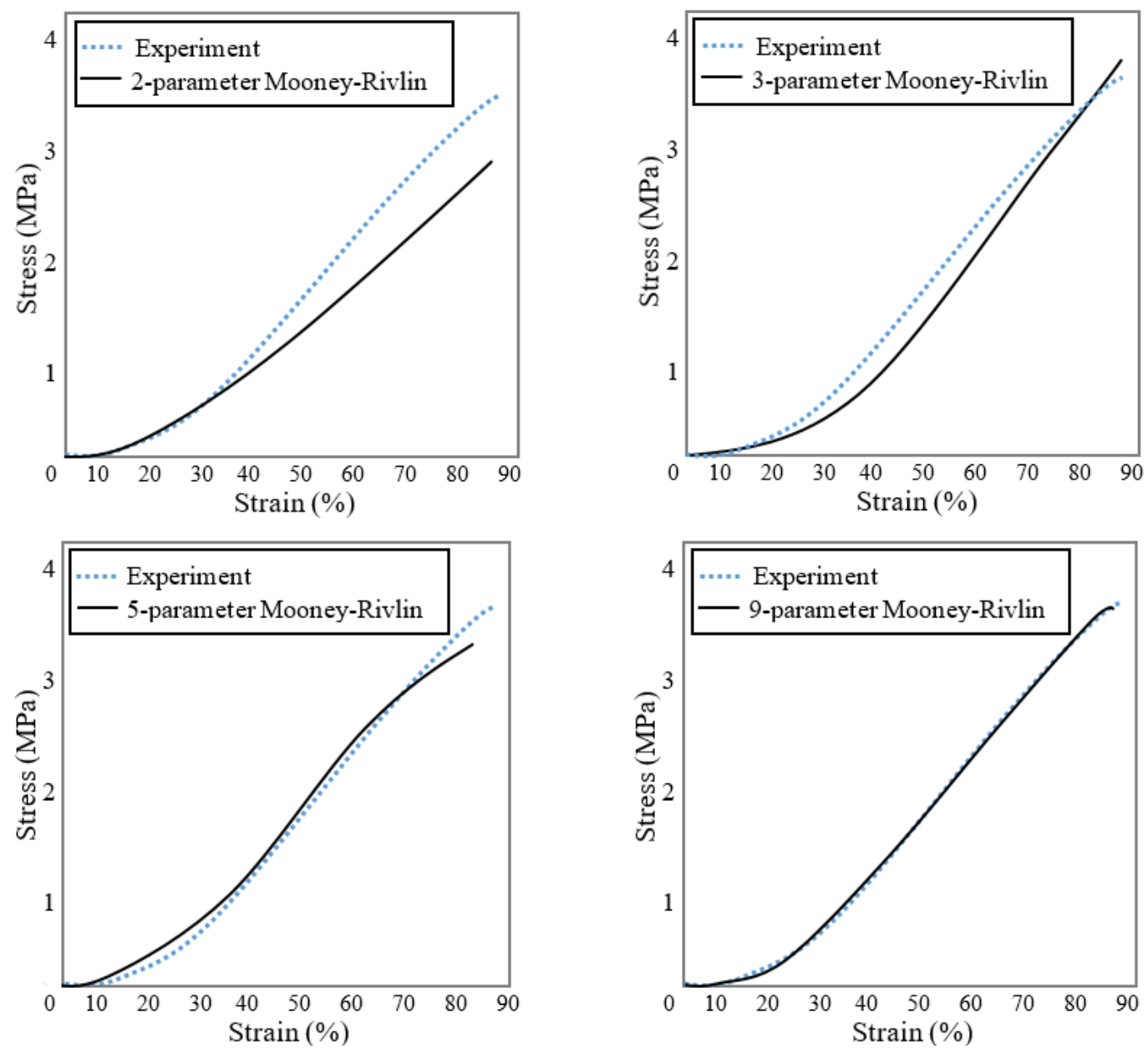

Fig. 11. Schematic Mooney-Rivlin Energy function curve fitting for hyperelastic materials.

A list of different constitutive models used in the FEA of 3D/4D-printed SPAs in terms of different materials is compiled in Table 3. A comparative study of experimental and FEA results, including the Ogden, Yeoh, Mooney Rivlin, and Arruda-Boyce models demonstrated the outperformance of the Ogden model for the actuator made from FDM 3D-printed TPU filament (Yap, $\mathrm{Ng}$, and Yeow 2016). In another study, where the four hyperelastic models, Arruda-Boyce, Neo-Hookean, Yeoh, Mooney-Rivlin, and Ogden utilized for the simulation of a 3D/4D-printed SPA using a thermoplastic elastomer, FilaFlex, it was reported that the Mooney-Rivlin model outperformed others (Hu 2019). The authors attributed the superior performance of the model to its ability to almost perfectly fit the experimental stress-strain curve with the smallest residual value. The combinations of the strain energy density function models were also utilized for the modeling of a composite 3D/4D-printed SPA where it was reported that the Ogden model was fitted for Dragon Skin-10 (Byrne et al. 2018) while the Polynomial model was selected for TPE (Ninjaflex, ink3D.ie, Dublin).

In a recent study, a new FEA method called "design and fabrication in the loop" was introduced to avoid extensive material tensile tests required for modelling the bending-type SPAs (Irawan, Ritonga, and Prastowo 2019) (highlighted in Fig. 12). The first order Yeoh model was employed with a focus on selecting the appropriate value of $C_{10}$ to obtain a closer prediction of experimental results. It was reported that the model and value of $C_{10}$ could not predict the behavior of SPAs with low deflections while the results were more accurate for bending angles 
more than $130^{\circ}$. A $3^{\text {rd }}$ order Yeoh model was also successfully implemented for the bending modeling of TPU made 3D/4D-printed SPA (Rosalia, Ang, and Yeow 2018). Different material models, including Mooney-Rivlin, Neo-Hookean, and Ogden were developed to obtain maximum consistency with experimental results of an additively manufactured SPA made of TPU (Dilibal, Sahin, and Celik 2018). It was reported that the Ogden material model predicted the closest result to the experimental bending motion of the SPA compared to the others in that study.

For modeling of a 3D/4D-printed SPA made from TangoBlack material, a three-parameter Mooney-Rivlin model was adopted to simulate the nonlinear elasticity. The model successfully implemented to identify the coefficients through the experimental results of plane strain tension tests. A 5-parameter Mooney-Rivlin model was also successfully utilized in ANSYS for simulating both bending angle and blocking/tip force of a fin-ray printed SPA made of NinjaFlex (Tawk, Gao, et al. 2019). Tetrahedral mesh elements were used in the study by defining the contact pair between the adjacent walls during the blocking/tip force simulation. Adding the passive fin-ray structure to the design was confirmed to improve the gripping capabilities of the gripper via both FEA and experiment results. The Mooney-Rivlin was also successfully used for FEA of 3D/4D-printed SPAs made via DLP technology (Zhang, Ng, et al. 2019) (Hingorani et al. 2019).

Overall, there is not one recipe serve all Energy models that could be recommended for FEA of the 3D/4D-printed SPAs. However, here we have summarized our investigations in terms of different models successfully achieved in terms of various hyperelastic materials used in the fabrications of these SPAs in Table 3. It is also shown that the Ogden has worked better than others in the FDM 3D-printed TPU filament SPA (Yap, Ng, and Yeow 2016) the first order fitting model of Yeoh was not successful in predicting the bending angle of less than $130^{\circ}$ with the same material. Ogden has also modeled the SPAs made from Dragon Skin-10 (Byrne et al. 2018) while the Polynomial model perfectly worked for TPE (Ninjaflex, ink3D.ie, Dublin). Also, Mooney-Rivlin was the model that outperformed others for the FilaFlex (Hu 2019).
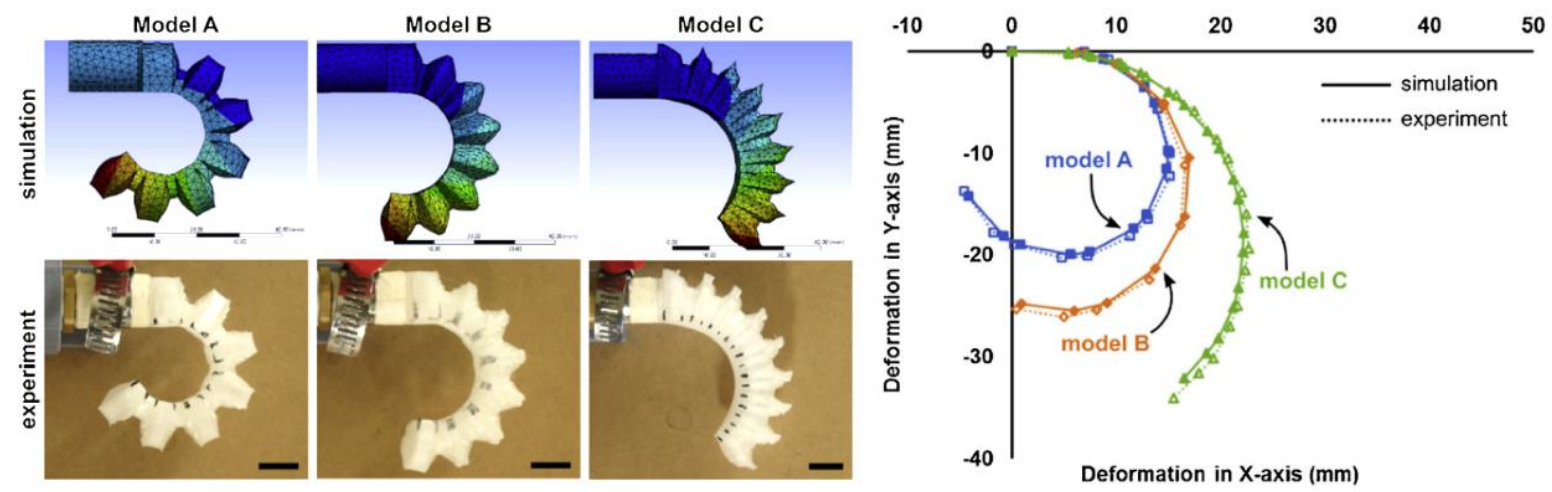

Fig. 12. The FEA of 3D/4D-printed SPAs with a different design, reproduced from (Irawan, Ritonga, and Prastowo 2019) with the permission of Elsevier. 
Table 3. The FEA models used for 4D printing bending-type SPAs.

\begin{tabular}{llll} 
Model & FE Platform & Materials & Reference \\
\hline Mooney-Rivlin & ABAQUS & EAA and AUD & (Patel et al. 2017) \\
& ABAQUS & TPU, NinjaFlex (NinjaTek, PA) & (Yap, Ng, and Yeow 2016) \\
& ABAQUS & TangoPlus and Ebecryl 113 & (Zhang, Ng, et al. 2019) \\
& ABAQUS & TangoPlus, EAA and AUD & (Hingorani et al. 2019) \\
& ANSYS & Tangle Black (Connex3 Object350 Stratasys, Co., Ltd.) & (Wang, Ge, et al. 2017) \\
& ANSYS & TPU, NinjaFlex (NinjaTek, PA) & (Tawk, Gao, et al. 2019) \\
& Marc Mentat & TPU & (Dilibal, Sahin, and Celik 2018) \\
\hline Neo-Hookean & ABAQUS & Tangoplus FLX 930 and Rhodamine B & (Ge18) \\
& ABAQUS & RTV-2 silicone rubber and PA12 nylon & (Ding et al. 2019) \\
& Marc Mentat & TPU & (Dilibal, Sahin, and Celik 2018) \\
\hline Linear & ANSYS & Elastomeric precursor (EP; Spot-E resin, Spot-A Materials, Inc.) & (Peele et al. 2015) \\
\hline Polynomial & ABAQUS & TPU (Ninjaflex, ink3D.ie, Dublin) & (Yap, Ng, and Yeow 2016) \\
& ABAQUS & TPE and Dragon Skin-10 & (Byrne et al. 2018) \\
& ABAQUS & VeroWhite+ and Agilus30 (Stratasys, Co., Ltd.) & (Du Pasquier et al. 2019) \\
\hline Yeoh & ANSYS & TPU (eSUNeFlex, Shenzhen Esun Industrial Co., Ltd.) & (Irawan, Ritonga, and Prastowo 2019) \\
& ABAQUS & TPU, NinjaFlex (NinjaTek, PA) & (Yap, Ng, and Yeow 2016) \\
& ABAQUS & TPU, NinjaFlex (NinjaTek, PA) & (Rosalia, Ang, and Yeow 2018) \\
& ABAQUS & TPU, NinjaFlex (NinjaTek, PA) & (Keong and Hua 2018) \\
& ABAQUS & Silicone (Keyence@ Agilista 3200®) & (Manns, Morales, and Frohn 2018) \\
& ABAQUS & TPU, NinjaFlex (NinjaTek, PA) & (Yap, Ng, and Yeow 2016) \\
& ABAQUS & Silicone (Dragon Skin 10, Smooth-On Inc., Macungie, PA) & (Byrne et al. 2018) \\
\hline Ogden & ABAQUS & TPU (NinjaFlex, NinjaTek) & (Hainsworth et al. 2020) \\
& Marc Mentat & TPU & (Dilibal, Sahin, and Celik 2018) \\
& ABAQUS & TPU, NinjaFlex (NinjaTek, PA) & (Yap, Ng, and Yeow 2016) \\
\hline Arruda-Boyce & &
\end{tabular}




\section{Control of 4D-printed Bending-type Soft Pneumatic Actuators 5.1. Integration of Sensors}

The inherent flexibility and compliance morphology of 3D/4D-printed SPAs require the integration of non-conventional sensors for their control. Thus, there have been investigations on the development of stretchable curvature sensors with minimal impact on the compliance of SPAs. However, due to the nonlinear behavior of the elastomeric materials in different environmental conditions, such as temperature and humidity changes, other types of chemical and biological sensors are required too. Feedforward and feedback controllers were applied for the control of 3D/4D-printed SPAs (George Thuruthel et al. 2018). Providing feedback information required for closed-loop control of SPAs demand availability of sensory feedback instruments that could be realized either using external measurement devices, such as camera (Marchese, Katzschmann, and Rus 2014) electromagnetic sensor (Wang et al. 2014), optical sensor (Zhuang et al. 2018; Darzi and Park 2017) or integrated flexible sensors (Khosravani and Reinicke 2020a) that do not compromise the conformability of the SPAs (Low et al. 2017) (Elgeneidy et al. 2018).

To facilitate the use of 3D/4D-printed SPAs in real-world applications, curvature or strain sensing is required in the closed-loop control algorithm for measuring the bending. The curvature sensors were integrated into 3D/4D-printed SPAs for grasping and trajectory tracking of the actuators during bending. These sensors have been fabricated via various 3D printing approaches, such as DIW, FDM, and PolyJet, to be directly integrated into the SPAs. The integrated flexible sensors could be in the form of adding conductive fillers such as carbon nanotube (CNT) (Giffney et al. 2016) and multi-walled carbon nanotube (MWCNT) (Lee et al. 2018 ) to elastomer materials with the possibility of 3D printing. To find more understanding of the grasping dynamics between a SPA gripper and deformable objects, a curvature resistive sensor (Spectra Symbol) was integrated to the bottom of the gripper (Wang and Hirai 2016). The slippage detection of the object during lift was achieved using the inserted flexible sensor. An integrated sensor for 3D/4D-printed SPA was directly fabricated using a multi-material 3D printer (Objet350, Stratasys Ltd., USA) and rubber-like, AgilusBlack, material for both actuation and self-power triboelectric curvature sensing (Zhu et al. 2020). The sensor was reported to measure a curvature up to $8.2 \mathrm{~m}^{-1}$ in a low-frequency rate of $0.06 \mathrm{~Hz}$. A flexible sensor was directly 3D-printed and embedded into soft pneumatic gripper to enhance its both exteroceptive and interoceptive capabilities (Shih et al. 2019). The resistive sensor was cofabricated with the soft gripper using a commercial Connex3 Objet350 multi-material 3D printer and directly integrated into a pneumatic gripper. The black resin containing carbon particles was used to produce conductivity required for the sensor traces. The tests demonstrated the capability of the sensor to differentiate between objects after grasping by the actuator.

To extend the capabilities of SPAs, tactile force-sensing could be employed for closed-loop control of SPAs for safe manipulation, shape recognition, texture Discrimination, and grasping in diverse applications (Sonar et al. 2020; Zhou et al. 2020). However, the state-of-the-art is to entirely automate the integration of sensors and actuators using readily available 3D printers (Hohimer et al. 2020; Elgeneidy et al. 2018). 
Multiple haptic capabilities, including thermoceptive and proprioceptive sensing, were simultaneously embedded in a 3D/4D-printed somatosensitive SPA (Truby et al. 2018). Conductive ionogel was used as conductive ink showing an acceptable level of longevity and low hysteresis (Truby et al. 2019; Truby et al. 2018; Zolfagharian, Kaynak, and Kouzani 2019). An ionically conductive hydrogel was one of the earliest sensors made by the DIW technique for measuring the tactile external stimuli based on the capacitive principle (Robinson et al. 2015). The so-called capacitive skin was able to detect a compressive force of $2 \mathrm{~N}$ for feedback control purposes. In another study, an inexpensive, flexible force sensor was devised for measuring the grasping forces exerted on the actuators to provide feedback information (Low et al. 2017). The flexible force sensor was made of a composite of electrodes and piezoresistive fabric (LW-SLPA, Eeonyx, CA) through printing conductive ink on the polyethylene substrate (AgIC, Japan). A self-sensing SPA was developed by common FDM technology and using two materials, TPU (NinjaFlex, NinjaTek) and ProtoPasta, which is carbon black (CB) doped polylactic acid (PLA), with no human intervention during the fabrication (Hainsworth et al. 2020). The actuator has shown acceptable compliance for grasping while providing feedback information. In other studies, both pressure and position sensors were integrated into SPAs made of TPU (Yang and Chen 2017). The sensing principle was based on the volume resistivity changes of conductive elastomers made by the addition of CB conductive fillers to TPU.

The embedded strain sensors made of liquid metals, such as eutectic gallium-indium (EGaIn), are also common in highly compliant soft gripper applications where extremely high deformations accompanying actuation can damage conventional sensing devices such as strain gauges (Fang et al. 2019; White, Case, and Kramer 2017). The optical Fiber Bragg Grating (FBG) sensors could also be used for imparting tactile capability in SPAs (Bogue 2016; He, Zhu, et al. 2018) due to their thin geometry, low hysteresis, and high speed and robustness. These sensors detect strain by measuring shifts in the wavelength of the reflected light. But, their drawback is limitations in large deformations. The Hall Effect magnetic field sensors have been developed by embedding the magnetic particles into soft materials, such as silicon. These types of sensors could be suitable for 3D/4D-printed SPAs due to their remote sensing of curvature (Ozel et al. 2016; Ozel et al. 2015) as well as accurate contact force measurements (Mirzanejad and Agheli 2019; Wang et al. 2016).

Environmental sensors for measuring the temperature (Yamaguchi, Kashiwagi, et al. 2019), humidity (Maldonado et al. 2019), and other chemical substances (Raman, Cvetkovic, and Bashir 2017) could be integrated to 3D/4D-printed SPAs for the purposes, such as stiffness variable adjustments and control of uncertain conditions.

Different types of soft sensors suitable for 3D/4D-printed bending-type SPAs are listed in Table 4. The selection criteria of the sensors could be varied based on different applications. However, in general, the DIW and FDM printers have shown superior performance due to resistive and capacitive sensors being directly $3 \mathrm{D}$-printed and integrated to the SPAs and also allowing for the use of a wide range of materials and composites. The DIW of conductive ionogel has shown promising longevity and low hysteresis performance. The strain sensors 3Dprinted by EGaIn are mainly suitable for extremely high deformations actuation. The FBG sensors, though needing further assembly requirements, are low in hysteresis and have robust functionalities. Also, the Hall Effect magnetic field sensors are highly accurate for contact force measurements for remote sensing capability. 
(a)

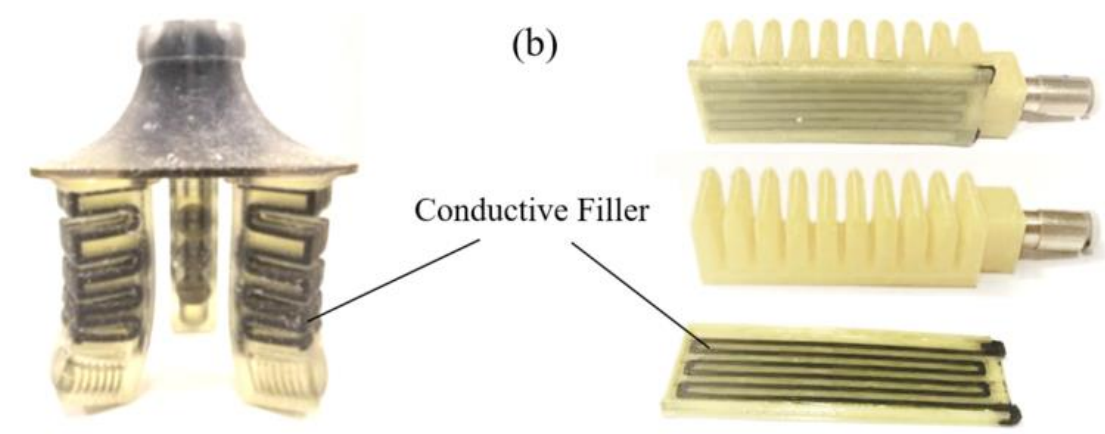

(c)

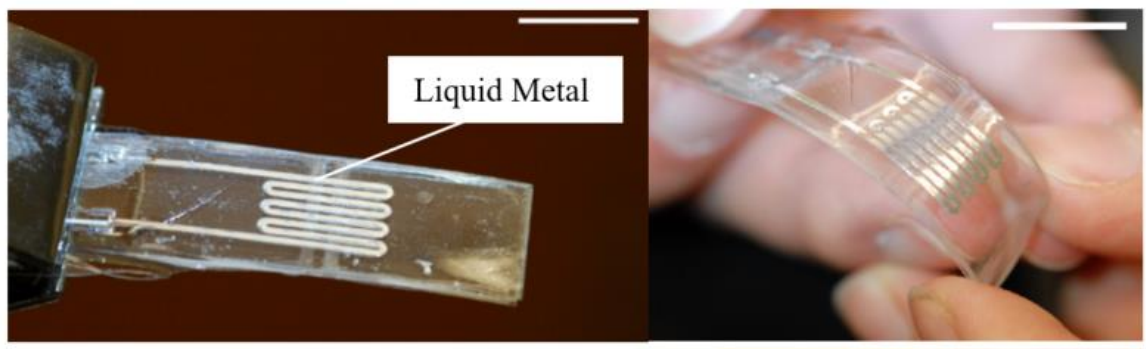

(d)

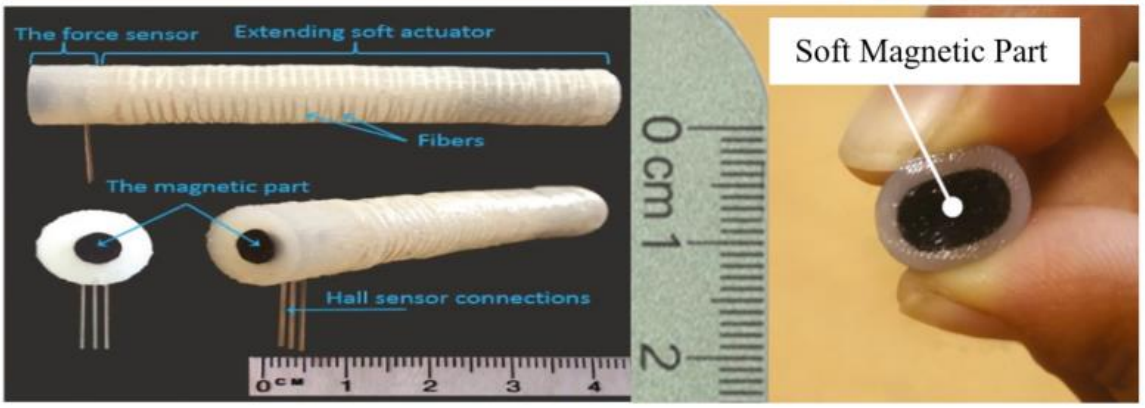

Fig. 13. Integrating sensors into 3D/4D-printed SPAs (a) conductive filler Polyjet 3D-printing, reproduced from (Shih et al. 2019) with permission of Frontiers under the terms of the Creative Commons CC BY license; (b) conductive filler FDM 3D printing, reproduced from (Elgeneidy et al. 2018) with permission of Frontiers under the terms of the Creative Commons CC BY license; (c) liquid metal DIW 3D printing (the scale is $6.25 \mathrm{~mm}$ ), reproduced from (White, Case, and Kramer 2017) with permission of Elsevier; (d) soft magnetic force sensor DIW 3D printing, reproduced from (Mirzanejad and Agheli 2019) with permission of Elsevier. 
Table 4. Different types of soft sensors suitable for 3D/4D-printed bending-type SPAs.

\begin{tabular}{|c|c|c|c|}
\hline Types & Applications & Mechanisms & Materials \\
\hline \multirow{11}{*}{ Curvature/Strain } & Grasping, & Capacitive (Frutiger et al. 2015) & Silicone \\
\hline & Trajectory tracking & Resistive (Eijking, Sanders, and Krijnen 2017) & TPU \\
\hline & & Resistive (Elgeneidy et al. 2018) & TPU/PLA \\
\hline & & Resistive (Yang and Chen 2017) & TPU/CB \\
\hline & & Liquid metal (White, Case, and Kramer 2017) & Magnetite/ABS \\
\hline & & Liquid metal (Fang et al. 2019; Yuan et al. 2019) & EGaIn/Ecoflex0050 \\
\hline & & Hall effect (van Tiem et al. 2015) & Magnet/plastic laminate \\
\hline & & Hall effect (Ozel et al. 2016; Ozel et al. 2015) & Magnet/silicone \\
\hline & & Optical waveguide (Wolfer et al. 2016) & InkOrmo/InkEpo \\
\hline & & Optical FBG (Wang et al. 2019) & Polyimide \\
\hline & & $\begin{array}{l}\text { Eddy current (Wallin, Pikul, and Shepherd 2018; Jeranče, Bednar, } \\
\text { and Stojanović 2013; Li et al. 2018) }\end{array}$ & Copper/ABS \\
\hline \multirow[t]{10}{*}{ Tactile Force } & Safe manipulation, & Capacitive (Laszczak et al. 2015) (Robinson et al. 2015) & Ionic gel \\
\hline & Shape sensing, & Capacitive (Ou et al. 2016) & TPU \\
\hline & Texture Discrimination, & Resistive (Lee et al. 2018) & Tango+/MWCNT \\
\hline & Different shapes grasping & Resistive (Yamaguchi, Arie, et al. 2019) & Ag/conductive thread \\
\hline & & Piezoelectric (Yoshida et al. 2018) & Tango Black \\
\hline & & Piezoresistive (Mousavi et al. 2018) & Cilia \\
\hline & & Piezoresistive (Sankar et al. 2019) & Fabric \\
\hline & & Liquid metal (Lopes et al. 2018) (Fang et al. 2019) & EGaIn \\
\hline & & Hall effect (Mirzanejad and Agheli 2019; Wang et al. 2016) & ABS/magnet \\
\hline & & Optical FBG (Lin et al. 2016) & $\mathrm{ABS} / \mathrm{FBG}$ \\
\hline \multirow[t]{2}{*}{ Temperature } & Variable stiffness & Capacitive (Wickberg et al. 2015) & Nanocrystals \\
\hline & & Thermistor (Yamaguchi, Kashiwagi, et al. 2019) & Polyethylene terephthalate \\
\hline Humidity & Classification & Solvatochromic (Maldonado et al. 2019) & 1D coordination polymer \\
\hline \multirow[t]{2}{*}{ Environmental } & Detection & Electrochemical (Raman, Cvetkovic, and Bashir 2017) & PEGDA \\
\hline & & Electrochemical (Gaál et al. 2018) & Graphene/PLA \\
\hline
\end{tabular}




\subsection{Data-driven machine learning modeling and control}

The conventional approaches used for analytical kinematic modeling of 3D/4D-printed SPAs, including the piecewise constant curvature (PCC) (Webster III and Jones 2010), and other discrete rigid elements models (Zolfagharian et al. 2018; Zhong, Hou, and Dou 2019) were developed based on assumptions that are only valid for specific simplified designs of the actuators mainly used in the structured environments. However, these models are not favorable for dealing with the nonlinearity and time-variable parameters associated with 3D/4D-printed SPAs in their practical applications in unstructured environments. The FEA also has some intrinsic drawbacks, such as imprecise constitutive law of hyperelastic materials and computational cost which are unsatisfactory for the controller design. Therefore, the use of machine learning techniques could be a more effective and reasonable choice for kinematic modeling of these systems.

The dynamics modeling of SPAs is even more complex than kinematics where data-driven modeling could be applied for learning dynamic models (Thuruthel et al. 2018; Zolfagharian et al. 2020; Zolfagharian, Kaynak, and Kouzani 2019). To include nonlinearity and hysteresis that occur in unconstrained dynamic environments into the design of 3D/4D-printed SPAs, the constitutive law of material behavior, particularly a modified Kelvin-Voigt, model was incorporated into dynamic modeling to embody the visco-hyperelasticity of SPAs (Mustaza et al. 2019). The proposed model was validated using experimental data for accurately reflecting the nonlinear and hysteresis behavior of the SPA, which could be used for model-based feedback control.

The data-driven machine learning approach was also previously used for modeling the nonlinearity of the SPAs arising from variation in solenoid valve flow rates during pressurization and depressurization (Mohamed et al. 2020; Mosadegh et al. 2014). Such non-linearity in addition to the multi-material combination in the manufacturing, and effects of other embedded components in the body of SPAs, could not be addressed merely by FEA, thus causing difficulty in the accurate bending control of 3D/4D-printed SPAs in practice. Therefore, higher control accuracy could be achieved in the empirical model using real-life data and integrated sensing capabilities of SPAs compared to FEA and analytical models.

The input pressure flow rate and its duration are the controllable parameters that could be regulated based on the desired output, which is the end-point position and tip force of the actuator. Hence, the model outlining the relationship between the input pressure and the bending angle response should be developed first. Thus, the pressure sensors connected to the pneumatic supply tubes and the integrated sensors are required for developing the data-driven model. One drawback of this approach is the high feedback noise level borne during the data acquisition of input pressure. This was significantly rectified by introducing a fixed volume syringe pneumatic air tank and a pneumatic resistor to damp the undesired oscillations as well as by applying a moving average digital filter (Elgeneidy, Lohse, and Jackson 2018). Having developed the data-driven model to estimate the bending angle based on the input pressure measurements, the closed-loop controller is required to meet the target bending angle.

The supervised learning, including linear regression and artificial neural network (ANN), models were commonly investigated on learning the inverse kinematics of SPAs (Elgeneidy, Lohse, and Jackson 2016; George Thuruthel et al. 2017; Thuruthel et al. 2019). The kinematics of SPAs, as discussed in the earlier section, is highly nonlinear in an unstructured environment 
due to hyperelastic material properties and design geometry. Thus, the ANN models could be employed to approximate the Jacobian function of SPAs (George Thuruthel et al. 2018). A comparison study of using both linear regression and ANN for predicting the bending angle of the SPA end-point was investigated (Elgeneidy, Lohse, and Jackson 2018). The authors reported that the ANN used in the study was able to reflect the nonlinearity in the response of SPA with higher accuracy than the linear regression model. The model used in their work was further utilized for finding the PID gains of the closed-loop controller to handle the external disturbances during the operation of the SPA. However, due to the nonlinearity of the 3D/4Dprinted SPAs, the conventional PID controller might not be an ideal choice for dealing with the uncertainties of the system. Hence, cascade controllers with the incorporation of machine learning algorithms to tune the PID gains could be adopted for improving the accuracy of the end-point position tracking of SPA (Zhao, Zhong, and Fan 2015; Fan et al. 2015) (Zolfagharian, Valipour, and Ghasemi 2016). A nonlinear autoregressive-exogenous (NARX) observer, using wavelet and sigmoid networks was also developed in the form of an Extended Kalman Filter to predict the behavior of the SPA operating in an unstructured environment with minimum error (Loo et al. 2019). The schematic workflow for the data-driven modeling and control of 3D/4D-printed SPAs is illustrated in Fig. 14.

(a)

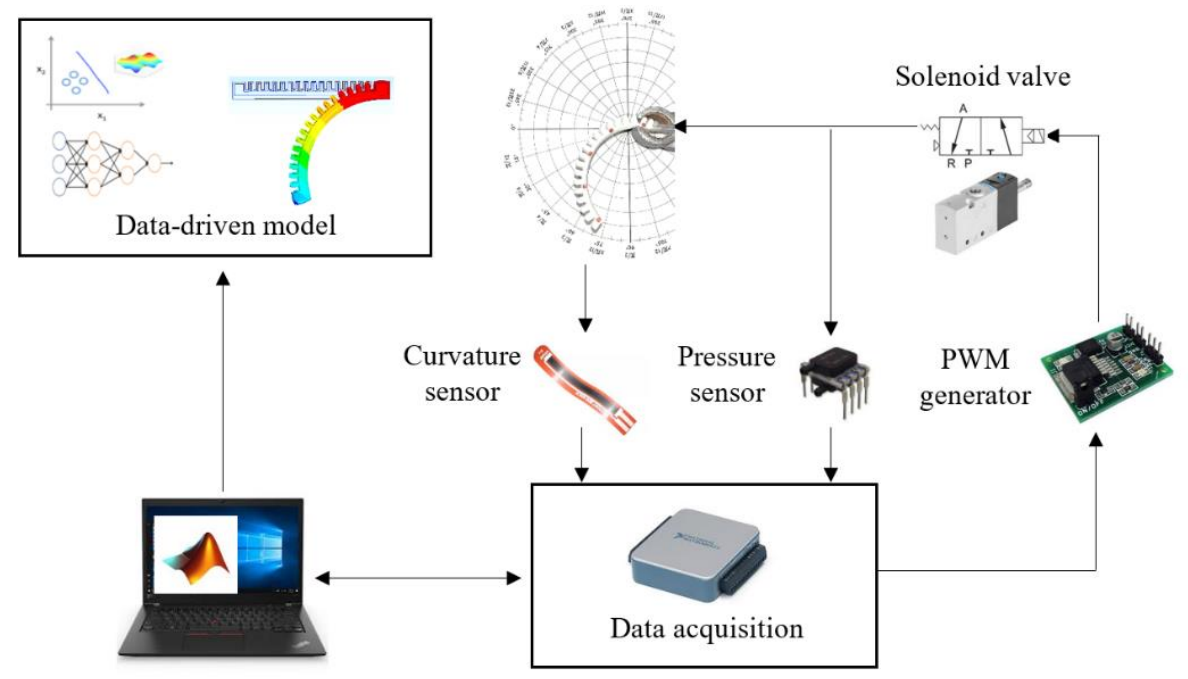

(b)

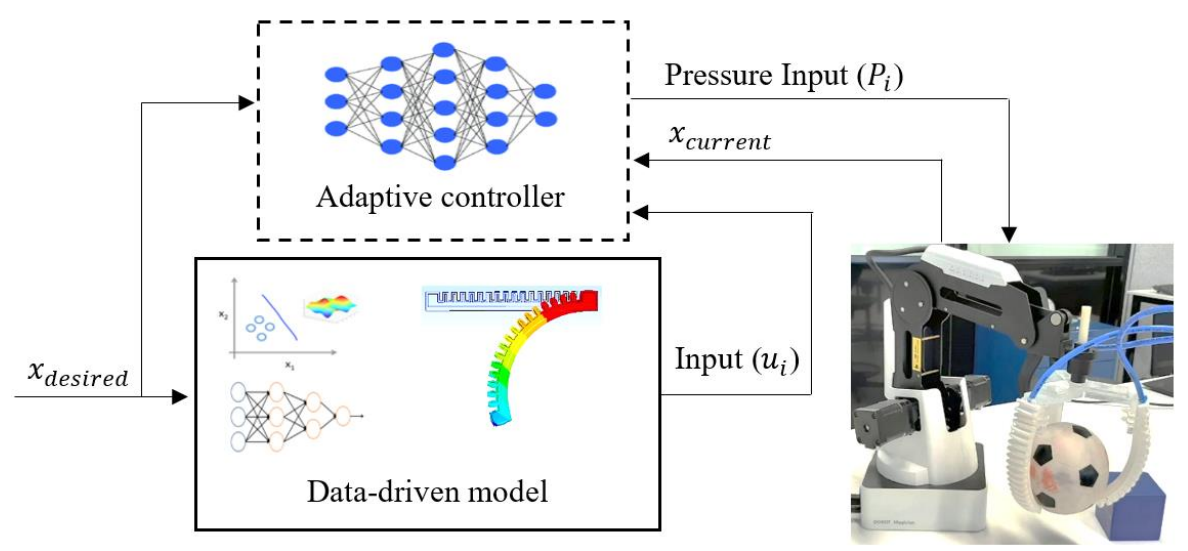

Fig. 14. (a) Data-driven modeling and, (b) closed-loop adaptive control of 3D/4D-printed SPAs. 


\section{Discussions, Challenges, and Future Directions}

There is a range of commercially available photocurable elastomers, including EPU 40 (Carbon), Tangoplus (Stratasys), Formlab Flexible (Formlab), and Spot-E Elastic (SpotAMaterials). The reviewed custom-made photo resins could be studied for an optimized mass ratio of the photo absorber and the resin to obtain customized and enhanced mechanical properties for 3D/4D printing of SPA via the DLP method (Ge et al. 2018).

One major concern of bending SPA is the balling effect of the lower chamber, which occurs due to abrupt change of the contour shape and may lead to an exertion of excessive pressure and even cause bursting of the actuator. The balling effect in a soft robot essentially wastes energy on radial inflation when the energy needs to be exercised on longitudinal inflation. Using multi-material 3D/4D printing is a proven way to deal with this problem in the SPA (Wang, Ge, et al. 2017). In this approach, a material with a higher modulus of elasticity is printed at the bottom layer of the SPA without compromising the bottom wall thickness. However, reducing the balling effect comes at the expense of the magnitude of the bending angle.

Miniaturizing and scaling down the 3D/4D printed SPAs are highly desirable, particularly by adding the micrometer-size functionalities for practical applications in the manipulation of microscale delicate objects, e.g. cells. Yet, such feature should be scalable in all the key components of the SPAs, including the integrated sensors (Khosravani and Reinicke 2020a), flexible electronics (Nasiri and Khosravani 2020), and controllers (Zolfagharian et al. 2020). However, miniaturizing the SPAs is constrained by the resolution of $3 \mathrm{D}$ printers and challenges in $3 \mathrm{D}$ printing such as avoidance of microscale voids and channels.

The power source of 3D-printed SPAs is a major point of emphasis using compressors, particularly for wearable SPAs in performing high mobility demand tasks such as medical applications and rehabilitation (Adami and Seibel 2019). Such applications require small-scale pressure generation systems as compact, lightweight, and low noise as possible where alternative methods such as onboard pneumatic pressure generation methods using chemical reaction-based sources or combustible fuels could be considered instead of bulk and heavy industrial-sized compressors (Walker et al. 2020). However, various factors, including the energy density, flow capacity, and safety are implicated in the selection of the most suited pressure generation method. For instance, it was reported that the battery-powered micro compressors provide relatively high capacity, but maximum pressure and flow rate are lower than the fluidic compressed tanks for soft robotics applications (Wehner et al. 2014).

Compressors are often used as the power source of 3D-printed SPAs, particularly for wearable SPAs in performing tasks that demand high mobility such as medical applications and rehabilitation (Adami and Seibel 2019). Such applications require small-scale pressure generation systems to be as compact, lightweight, and low noise as possible for which alternative methods such as on-board pneumatic pressure generation methods using chemical reaction-based sources or combustible fuels could be considered instead of bulk and heavy industrial-sized compressors (Walker et al. 2020). However, various factors, including the energy density, flow capacity, and safety are implicated in the selection of the most suitable pressure generation method. For instance, it was reported that the battery-powered microcompressors provided relatively high capacity, but their maximum pressure and flow rate were lower than the fluidic compressed tanks for soft robotics applications (Wehner et al. 
2014). Compact 3D/4D-printed SPAs could be developed with applications in soft mobile robots. The energy source of these printed soft robots could be supplied using onboard pneumatic pressure generation methods, such as microprocessors, pressurized gas tanks, chemical reactions, and phase change at the triple point which are shown to generate up to 500 $\mathrm{kPa}$ input pressure (Adami and Seibel 2019).

One of the important conditions for the sustainability of a product is the ability of the material used to be recycled at any time during its life cycle, such as during manufacturing, during use, and after product life. These recycling stages could be considered so that 3D-printed SPAs could be integrated into the recycling process (Nguyen and Seibel 2019). 3D/4D printing of custom-made SPAs could be justified in terms of their environmental impact due to utilization of green and recyclable materials, optimization of processing parameters, such as layer thickness and porosity during the $3 \mathrm{D}$ printing process, and to their reduced carbon-foot print and waste (Khosravani and Reinicke 2020b).

Out-of-plane deformation is understood as a drawback of current 3D/4D-printed SPAs. The twist-coupled bending or sideward slipping of SPAs during operation leads to unstable grasping. This issue could be further investigated in 4D printing with more consideration in the design and also on the localized stiffening of the structure of the SPA (Scharff et al. 2019).

A novel 3D printing manufacturing method was introduced with the incorporation of fiber weaving into an elastomeric material extrusion (Stalin et al. 2019). This provides a new manufacturing path for 4D printing a novel type of reinforced SPA that could be a mixture of both bellow-type and embedded fiber into the matrix of 3D-printed elastomer.

Recently a SPA was 4D-printed, which operates based on the self-exciting vibration principles. This actuator has shown vibrating capabilities without needing any valve which simplifies the structure for wider applications. The self-excitation feature of this actuator will further simplify the control and drive of 3D/4D-printed bending-type SPAs in mobile soft robots and microfluidics (Tani et al. 2020).

The grasping adaptability and stability of the 3D/4D-printed SPA could be further enhanced by introducing the variable stiffness capabilities by various means such as self-locking mechanism (Guo et al. 2020) or multi-material fabrication (Al-Rubaiai et al. 2019; Zhu et al. 2019; Du Pasquier et al. 2019). While the self-locking mechanism provides energy-saving and less valve control, a combination of 3D-printed sensors and multi-material fabrication using SMPs could be implemented to develop variable stiffness control of 3D/4D-printed SPAs (AlRubaiai et al. 2019; Fang et al. 2019).

The 3D/4D-printed SPAs are intrinsically soft and resilient to mechanical impacts, but at the expense of vulnerability to wear, puncture, and cut due to over pressurizing or contact with sharp objects. Although self-healing SPAs have already been investigated (Terryn et al. 2017), the 4D-printed SPAs with self-healing capability are rarely studied, and this could be a prospect future direction in 4D printing eco-friendly pneumatic soft robots. These SPAs could be recycled and reused while having a beneficial impact on the life span and reducing their ecological footprint.

The port-Hamiltonian model of 3D/4D-printed SPAs could bring more insights into the control-based modeling of these systems for practical applications in unstructured environments. A study of developing the port-Hamiltonian model for SPA has proved the 
efficacy of this approach for designing and controlling a more energy-efficient task-oriented SPA (Chun et al. 2019; Ghasemi, Xiao, and Gao 2019).

Further investigations on the behavior of 3D/4D-printed SPAs could be conducted for uncertain environments, such as amphibious for the under-water grasping ability for floating objects (Hao et al. 2017; Sun, Wang, and Zhu 2020; Zhang et al. 2020). This could be investigated further with advanced multimodal sensors (Chen et al. 2020) and electronic skin (e-skin) (Shih et al. 2020; Fu et al. 2020). The FEA could be used in kinematic model development of SPAs where an adequately large amount of data is required to train the ANN (Runge, Wiese, and Raatz 2017).

There is an opportunity for further investigations on the two-way fluid-structure interaction (FSI) meshless methods for accurately simulating the bending behavior of the 3D/4D-printed SPAs rather than merely one-way structural hyperelastic models (Moon et al. 2020). The early study has revealed that FSI outperformed the conventional structural FEA in nonlinear bending behavior of SPAs, particularly in the large deformation analysis (Moon et al. 2020).

Despite successful utilization of the data-driven controller in the closed-loop control of 3D/4Dprinted SPAs there are still challenges remaining in the high-frequency control bandwidth applications. The incorporation of the intrinsic controller via the morphological properties of voxels acting as zero-lag feedback controller during printing can broaden the control bandwidth for faster and more robust manipulations. The sparse sensing and uncertainty quantification could be incorporated via integration of 3D-printed sensors by optimising the locations of sensors to enhance controller performance. The incorporation of the morphological computation of materials into the controller account for the optimal placement of sensors to SPAs.

Currently, there are two different viewpoints on the control of soft manipulators (Shih et al. 2020). First, is the high-level control of the SPAs, like rigid manipulators and grippers, where a range of integrated soft sensors with low bandwidth, less than $50 \mathrm{~Hz}$, and higher bandwidth, up to $400 \mathrm{~Hz}$, are required to provide the tactile perception and vibration-induced information to avoid the slippage. The second view, however, is focusing more on proprioceptive feedback information on each volumetric pixel (voxel) of SPA to deliver low-level control tasks relying on the morphology of the SPA body, like the way octopus arm works. The final decision should be made to realize 3D/4D-printed SPAs that are safer for human-robots and are more aware of their environment.

\section{Conclusion}

The 3D-printed bending-type soft pneumatic actuators are the most common soft actuators that attracted the research community recently due to their unique characteristics such as custommade automated manufacturing, compliance, lightweight, and safety in human-robot interactions as well as the ability to handle delicate items. This study provided a comprehensive guide to fabrication, modeling, and control of 3D/4D-printed SPAs thus far developed. Different types of materials and 3D printing techniques used for developing these actuators were reviewed. The analytical kinematic modeling for predicting the bending curvature and blocking force of the 3D/4D-printed SPAs was described. The FEA for dynamic modeling of hyperelastic materials and various energy functions, which broadly applied for SPAs were 
discussed and the suitability of them was investigated based on common materials. The appropriate sensors that could be integrated into the 3D/4D-printed SPAs were analyzed. The control-based 4D printing of SPAs was presented and discussed. Finally, the current challenges were depicted and cutting-edge research avenues for starting new studies on 3D/4D-printed SPAs were highlighted.

\section{References}

Adami, Momme, and Arthur Seibel. 2019. "On-board pneumatic pressure generation methods for soft robotics applications." In Actuators, 2. Multidisciplinary Digital Publishing Institute.

Ainla, Alar, Mohit S Verma, Dian Yang, and George M Whitesides. 2017. 'Soft, rotating pneumatic actuator', Soft robotics, 4: 297-304.

Al-Rubaiai, Mohammed, Thassyo Pinto, Chunqi Qian, and Xiaobo Tan. 2019. 'Soft actuators with stiffness and shape modulation using 3D-printed conductive polylactic acid material', Soft robotics, 6: 318-32.

Alici, Gursel, Taylor Canty, Rahim Mutlu, Weiping Hu, and Vitor Sencadas. 2018. 'Modeling and experimental evaluation of bending behavior of soft pneumatic actuators made of discrete actuation chambers', Soft robotics, 5: 24-35.

Anver, Hisham MCM, Rahim Mutlu, and Gursel Alici. 2017. "3D printing of a thin-wall soft and monolithic gripper using fused filament fabrication." In 2017 IEEE International Conference on Advanced Intelligent Mechatronics (AIM), 442-47. IEEE.

Arruda, Ellen M, and Mary C Boyce. 1993. 'A three-dimensional constitutive model for the large stretch behavior of rubber elastic materials'.

Auysakul, Jutamanee, Nitipan Vittayaphadung, Sarawut Gonsrang, and Pruittikorn Smithmaitrie. 2020. 'Bending Angle Effect of theCross-Section Ratio for a Soft Pneumatic Actuator', International Journal of Mechanical Engineering and Robotics Research, 9.

Bartlett, Nicholas W, Michael T Tolley, Johannes TB Overvelde, James C Weaver, Bobak Mosadegh, Katia Bertoldi, George M Whitesides, and Robert J Wood. 2015. 'A 3D-printed, functionally graded soft robot powered by combustion', Science, 349: 161-65.

Batsuren, Khulan, and Dongwon Yun. 2019. 'Soft robotic gripper with chambered fingers for performing in-hand manipulation', Applied Sciences, 9: 2967.

Blatz, Paul J, and William L Ko. 1962. 'Application of finite elastic theory to the deformation of rubbery materials', Transactions of the Society of Rheology, 6: 223-52.

Bodaghi, M, AR Damanpack, and WH Liao. 2016. 'Self-expanding/shrinking structures by 4D printing', Smart Materials and Structures, 25: 105034.

Bodaghi, M, Damanpack, A.R. and Liao, W.H. 2017. 'Adaptive metamaterials by functionally graded 4D printing', Materials \& Design, 135: 26-36.

Bogue, Robert. 2016. 'Flexible and soft robotic grippers: the key to new markets?', Industrial Robot: An International Journal, 43: 258-63.

Boyce, Mary C. 1996. 'Direct comparison of the Gent and the Arruda-Boyce constitutive models of rubber elasticity', Rubber chemistry and technology, 69: 781-85.

Byrne, Oisín, Fergal Coulter, Mark Glynn, James FX Jones, Aisling Ní Annaidh, Eoin D O'Cearbhaill, and Dónal P Holland. 2018. 'Additive manufacture of composite soft pneumatic actuators', Soft robotics, 5: 726-36.

Cacucciolo, Vito, Jun Shintake, Yu Kuwajima, Shingo Maeda, Dario Floreano, and Herbert Shea. 2019. 'Stretchable pumps for soft machines', Nature, 572: 516-19.

Chen, Daobing, Qingping Liu, Zhiwu Han, Junqiu Zhang, HongLie Song, Kejun Wang, Zhengyi Song, Shifeng Wen, Yan Zhou, and Chunze Yan. 2020. '4D Printing Strain Self-Sensing and Temperature Self-Sensing Integrated Sensor-Actuator with Bioinspired Gradient Gaps', Advanced Science: 2000584.

Chen, Xiaoqian, Xiang Zhang, Hongwei Liu, and Yiyong Huang. 2019. 'Design and development of a soft robotic manipulator', International Journal of Mechanics and Materials in Design: 1-13. 
Chun, Ho-Tak D, Jamie O Roberts, Mohammed E Sayed, Simona Aracri, and Adam A Stokes. 2019. "Towards more Energy Efficient Pneumatic Soft Actuators using a Port-Hamiltonian Approach." In 2019 2nd IEEE International Conference on Soft Robotics (RoboSoft), 277-82. IEEE.

Connolly, Fionnuala, Conor J Walsh, and Katia Bertoldi. 2017. 'Automatic design of fiber-reinforced soft actuators for trajectory matching', Proceedings of the National Academy of Sciences, 114: 51-56.

Darzi, Milad, and Chanwoo Park. 2017. 'Optical distortion correction of a liquid-gas interface and contact angle in cylindrical tubes', Physics of Fluids, 29: 052004.

Davis, Steve. 2018. "pneumatic actuators." In Actuators, 62. Multidisciplinary Digital Publishing Institute.

Demir, Kahraman G, Zhizhou Zhang, Jehan Yang, and Grace X Gu. 'Computational and Experimental Design Exploration of 3D-Printed Soft Pneumatic Actuators', Advanced Intelligent Systems.

Dilibal, S, H Sahin, and Y Celik. 2018. 'Experimental and numerical analysis on the bending response of the geometrically gradient soft robotics actuator', Archives of Mechanics, 70: 391-404.

Ding, Longwei, Ning Dai, Xiaoming Mu, Shaohui Xie, Xu Fan, Dawei Li, and Xiaosheng Cheng. 2019. 'Design of soft multi-material pneumatic actuators based on principal strain field', Materials \& Design, 182: 108000.

Drotman, Dylan, Michael Ishida, Saurabh Jadhav, and Michael T Tolley. 2018. 'Application-driven design of soft, 3-D printed, pneumatic actuators with bellows', IEEE/ASME Transactions on Mechatronics, 24: 78-87.

Drozdov, AD. 2007. 'Constitutive equations in finite elasticity of rubbers', International Journal of Solids and Structures, 44: 272-97.

Du Pasquier, Cosima, Tian Chen, Skylar Tibbits, and Kristina Shea. 2019. 'Design and computational modeling of a 3D printed pneumatic toolkit for soft robotics', Soft robotics, 6: 657-63.

Eijking, Bram, Remco Sanders, and Gijs Krijnen. 2017. "Development of whisker inspired 3D multimaterial printed flexible tactile sensors." In SENSORS, 2017 IEEE, 1-3. IEEE.

Elgeneidy, Khaled, Niels Lohse, and Michael Jackson. 2016. 'Data-driven bending angle prediction of soft pneumatic actuators with embedded flex sensors', IFAC-PapersOnLine, 49: 513-20.

Elgeneidy, Khaled, Niels Lohse, and Michael Jackson. 2018. 'Bending angle prediction and control of soft pneumatic actuators with embedded flex sensors-a data-driven approach', Mechatronics, 50: 234-47.

Elgeneidy, Khaled, Gerhard Neumann, Michael Jackson, and Niels Lohse. 2018. 'Directly printable flexible strain sensors for bending and contact feedback of soft actuators', Frontiers in Robotics and Al, 5: 2.

Fan, Jizhuang, Jun Zhong, Jie Zhao, and Yanhe Zhu. 2015. 'BP neural network tuned PID controller for position tracking of a pneumatic artificial muscle', Technology and Health Care, 23: S231S38.

Fang, Xi, Zemin Liu, Yufei Hao, Hui Yang, Jiaqi Liu, Zhexin Xie, and Li Wen. 2019. "A Soft Actuator with Tunable Mechanical Configurations for Object Grasping Based on Sensory Feedback." In 2019 2nd IEEE International Conference on Soft Robotics (RoboSoft), 25-30. IEEE.

Forni, M, A Martelli, and A Dusi. 1999. 'Implementation and validation of hyperelastic finite element models of high damping rubber bearings', Constitutive models for rubber, 1: 237-47.

Frutiger, Andreas, Joseph T Muth, Daniel M Vogt, Yiğit Mengüç, Alexandre Campo, Alexander D Valentine, Conor J Walsh, and Jennifer A Lewis. 2015. 'Capacitive soft strain sensors via multicore-shell fiber printing', J Advanced Materials, 27: 2440-46.

$\mathrm{Fu}, \mathrm{Ya}$-Fei, Feng-Lian Yi, Jin-Rui Liu, Yuan-Qing Li, Ze-Yu Wang, Gang Yang, Pei Huang, Ning Hu, and Shao-Yun Fu. 2020. 'Super soft but strong E-Skin based on carbon fiber/carbon black/silicone composite: Truly mimicking tactile sensing and mechanical behavior of human skin', Composites Science and Technology, 186: 107910. 
Gaál, Gabriel, Tatiana Americo da Silva, Vladimir Gaál, Rafael Cintra Hensel, Lucas Rios Amaral, Varlei Rodrigues, and Antonio Riul Jr. 2018. '3D printed e-tongue', J Frontiers in chemistry, 6: 151.

Galley, Alexandre, George K Knopf, and Mohamed Kashkoush. 2019. "Pneumatic Hyperelastic Actuators for Grasping Curved Organic Objects." In Actuators, 76. Multidisciplinary Digital Publishing Institute.

Ge, Lisen, Longteng Dong, Dong Wang, Qi Ge, and Guoying Gu. 2018. 'A digital light processing 3D printer for fast and high-precision fabrication of soft pneumatic actuators', Sensors and Actuators A: Physical, 273: 285-92.

Gent, AN. 1996. 'A new constitutive relation for rubber', Rubber chemistry and technology, 69: 5961.

George Thuruthel, Thomas, Yasmin Ansari, Egidio Falotico, and Cecilia Laschi. 2018. 'Control strategies for soft robotic manipulators: A survey', Soft robotics, 5: 149-63.

George Thuruthel, Thomas, Egidio Falotico, Mariangela Manti, Andrea Pratesi, Matteo Cianchetti, and Cecilia Laschi. 2017. 'Learning closed loop kinematic controllers for continuum manipulators in unstructured environments', Soft robotics, 4: 285-96.

Ghasemi, Arman, Penghao Xiao, and Wei Gao. 2019. 'Nudged elastic band method for solid-solid transition under finite deformation', The Journal of Chemical Physics, 151: 054110.

Giffney, Tim, Mengying Xie, Aaron Yong, Andrew Wong, Philippe Mousset, Andrew McDaid, and Kean Aw. 2016. 'Soft pneumatic bending actuator with integrated carbon nanotube displacement sensor', Robotics, 5: 7.

Gorissen, Benjamin, Takuya Chishiro, Shuhei Shimomura, Dominiek Reynaerts, Michael De Volder, and Satoshi Konishi. 2014. 'Flexible pneumatic twisting actuators and their application to tilting micromirrors', Sensors and Actuators A: Physical, 216: 426-31.

Gorissen, Benjamin, Dominiek Reynaerts, Satoshi Konishi, Kazuhiro Yoshida, Joon-Wan Kim, and Michael De Volder. 2017. 'Elastic inflatable actuators for soft robotic applications', Advanced Materials, 29: 1604977.

Guo, Xin-Yu, Wen-Bo Li, Qiu-Hua Gao, Han Yan, Yan-Qiong Fei, and Wen-Ming Zhang. 2020. 'Selflocking mechanism for variable stiffness rigid-soft gripper', Smart Materials and Structures, 29: 035033.

Hainsworth, Travis, Lawrence Smith, Sebastian Alexander, and Robert MacCurdy. 2020. 'A Fabrication Free, 3D Printed, Multi-Material, Self-Sensing Soft Actuator', IEEE Robotics and Automation Letters.

Hao, Yufei, Tianmiao Wang, Ziyu Ren, Zheyuan Gong, Hui Wang, Xingbang Yang, Shaoya Guan, and Li Wen. 2017. 'Modeling and experiments of a soft robotic gripper in amphibious environments', International Journal of Advanced Robotic Systems, 14: 1729881417707148.

He, Yanlin, Lianqing Zhu, Guangkai Sun, Mingxin Yu, and Mingli Dong. 2018. 'Design, Measurement and Shape Reconstruction of Soft Surgical Actuator Based on Fiber Bragg Gratings', Applied Sciences, 8: 1773.

He, Yong, Luyu Zhou, Junfu Zhan, Qing Gao, Jianzhong Fu, Chaoqi Xie, Haiming Zhao, and Yu Liu. 2018. 'Three-dimensional coprinting of liquid metals for directly fabricating stretchable electronics', 3D Printing and Additive Manufacturing, 5: 195-203.

Hingorani, Hardik, Yuan-Fang Zhang, Biao Zhang, Ahmad Serjouei, and Qi Ge. 2019. 'Modified commercial UV curable elastomers for passive 4D printing', International Journal of Smart and Nano Materials, 10: 225-36.

Hohimer, Cameron J, Gayaneh Petrossian, Amir Ameli, Changki Mo, and Petra Pötschke. 2020. '3D Printed Conductive Thermoplastic Polyurethane/Carbon Nanotube Composites for Capacitive and Piezoresistive Sensing in Soft Pneumatic Actuators', Additive Manufacturing: 101281.

Holzapfel, GA. 2010. 'Some Forms of Strain-energy Functions', Nonlinear Solid Mechanics: $A$ Continuum Approach for Engineering; John Wlley \& Sons, LTD: Hoboken, NJ, USA: 238.

$\mathrm{Hu}$, Weiping. 2019. 'Flexible Fluidic Actuators for Soft Robotic Applications'. 
$\mathrm{Hu}$, Weiping, Weihua Li, and Gursel Alici. 2018. "3d printed helical soft pneumatic actuators." In 2018 IEEE/ASME International Conference on Advanced Intelligent Mechatronics (AIM), 95055. IEEE.

Hu, Weiping, Rahim Mutlu, Weihua Li, and Gursel Alici. 2018. 'A structural optimisation method for a soft pneumatic actuator', Robotics, 7: 24.

Irawan, Wira, Alim Syahirul Ritonga, and Aishah Prastowo. 2019. 'Design and fabrication in the loop of soft pneumatic actuators using fused deposition modelling', Sensors and Actuators A: Physical, 298: 111556.

Jeranče, Nikola, Nikola Bednar, and Goran Stojanović. 2013. 'An ink-jet printed eddy current position sensor', J Sensors, 13: 5205-19.

Ji, Myunggi, Qiang Li, In Ho Cho, and Jaeyoun Kim. 2019. 'Rapid Design and Analysis of Microtube Pneumatic Actuators Using Line-Segment and Multi-Segment Euler-Bernoulli Beam Models', Micromachines, 10: 780.

Keong, Benjamin Ang Wee, and Raye Yeow Chen Hua. 2018. 'A novel fold-based design approach toward printable soft robotics using flexible 3D printing materials', Advanced Materials Technologies, 3: 1700172.

Khondoker, Mohammad Abu Hasan, and Dan Sameoto. 2019. 'Direct coupling of fixed screw extruders using flexible heated hoses for FDM printing of extremely soft thermoplastic elastomers', Progress in Additive Manufacturing, 4: 197-209.

Khoo, Zhong Xun, Joanne Ee Mei Teoh, Yong Liu, Chee Kai Chua, Shoufeng Yang, Jia An, Kah Fai Leong, and Wai Yee Yeong. 2015. '3D printing of smart materials: A review on recent progresses in 4D printing', Virtual and Physical Prototyping, 10: 103-22.

Khosravani, Mohammad Reza, and Tamara Reinicke. 2020a. '3D-printed sensors: Current progress and future challenges', SENSORS AND ACTUATORS A-PHYSICAL, 305.

Khosravani, Mohammad Reza, and Tamara Reinicke. 2020b. 'On the environmental impacts of 3D printing technology', Applied Materials Today, 20: 100689.

Kim, Beomkeun, Seong Beom Lee, Jayone Lee, Sehyun Cho, Hyungmin Park, Sanghoon Yeom, and Sung Han Park. 2012. 'A comparison among Neo-Hookean model, Mooney-Rivlin model, and Ogden model for chloroprene rubber', International Journal of Precision Engineering and Manufacturing, 13: 759-64.

Kisner, Zack, Chris Szigeti, and David Leonardo. 2018. '3D Printed Soft Robotic Hand'.

Laszczak, Piotr, Liudi Jiang, Dan L Bader, David Moser, and S Zahedi. 2015. 'Development and validation of a 3D-printed interfacial stress sensor for prosthetic applications', J Medical engineering physics, 37: 132-37.

Lau, Hong Fai. 2019. '3D-Printed Inflatable Actuators: Design and Development of Soft Actuators for a Pneumatically-Actuated Soft Robotics Arm'.

Lee, Ju-Kyoung, Hyun-Hee Kim, Jae-Won Choi, Kyung-Chang Lee, and Suk Lee. 2018. 'Development of direct-printed tactile sensors for gripper control through contact and slip detection', International Journal of Control, Automation and Systems, 16: 929-36.

Leist, Steven K, and Jack Zhou. 2016. 'Current status of 4D printing technology and the potential of light-reactive smart materials as 4D printable materials', Virtual and Physical Prototyping, 11: 249-62.

Li, Bo, Lifan Meng, Hongyu Wang, Jing Li, and Chunmei Liu. 2018. 'Rapid prototyping eddy current sensors using 3D printing', J Rapid Prototyping Journal, 24: 106-13.

Liang, Xinquan, Yi Sun, and Hongliang Ren. 2016. 'A flexible fabrication approach toward the shape engineering of microscale soft pneumatic actuators', IEEE Robotics and Automation Letters, 2: $165-70$.

Lin, Ying-Kai, Tso-Sheng Hsieh, Liren Tsai, Shing-Hai Wang, and Chia-Chin Chiang. 2016. 'Using threedimensional printing technology to produce a novel optical fiber bragg grating pressure sensor', J Sensors Materials and Corrosion, 28: 389-94. 
Loo, Junn Yong, Kah Chun Kong, Chee Pin Tan, and Surya Girinatha Nurzaman. 2019. "Non-linear System Identification and State Estimation in a Pneumatic Based Soft Continuum Robot." In 2019 IEEE Conference on Control Technology and Applications (CCTA), 39-46. IEEE.

Lopes, Pedro Alhais, Hugo Paisana, Anibal T De Almeida, Carmel Majidi, and Mahmoud Tavakoli. 2018. 'Hydroprinted electronics: ultrathin stretchable Ag-In-Ga E-skin for bioelectronics and human-machine interaction', ACS applied materials \& interfaces, 10: 38760-68.

Low, Jin Huat, Wang Wei Lee, Phone May Khin, Nitish V Thakor, Sunil L Kukreja, Hong Liang Ren, and Chen Hua Yeow. 2017. 'Hybrid tele-manipulation system using a sensorized 3-D-printed soft robotic gripper and a soft fabric-based haptic glove', IEEE Robotics and Automation Letters, 2: 880-87.

Maldonado, Noelia, Verónica G Vegas, Oded Halevi, Jose Ignacio Martínez, Pooi See Lee, Shlomo Magdassi, Michael T Wharmby, Ana E Platero-Prats, Consuelo Moreno, and Félix Zamora. 2019. '3D Printing of a Thermo-and Solvatochromic Composite Material Based on a CU (II)Thymine Coordination Polymer with Moisture Sensing Capabilities', J Advanced Functional Materials: 1808424.

Manns, Martin, Jorge Morales, and Peter Frohn. 2018. 'Additive manufacturing of silicon based PneuNets as soft robotic actuators', Procedia CIRP, 72: 328-33.

Marchese, Andrew D, Robert K Katzschmann, and Daniela Rus. 2014. "Whole arm planning for a soft and highly compliant 2d robotic manipulator." In 2014 IEEE/RSJ International Conference on Intelligent Robots and Systems, 554-60. IEEE.

Marckmann, Gilles, and Erwan Verron. 2006. 'Comparison of hyperelastic models for rubber-like materials', Rubber chemistry and technology, 79: 835-58.

Mirzanejad, Hossein, and Mahdi Agheli. 2019. 'Soft force sensor made of magnetic powder blended with silicone rubber', Sensors and Actuators A: Physical, 293: 108-18.

Mishra, Anand K, Thomas J Wallin, Wenyang Pan, Patricia Xu, Kaiyang Wang, Emmanuel P Giannelis, Barbara Mazzolai, and Robert F Shepherd. 2020. 'Autonomic perspiration in 3D-printed hydrogel actuators', Science Robotics, 5.

Mohamed, Mahmoud H, Soha H Wagdy, Mostafa A Atalla, Aliaa Rehan Youssef, and Shady A Maged. 2020. 'A proposed soft pneumatic actuator control based on angle estimation from datadriven model', Proceedings of the Institution of Mechanical Engineers, Part H: Journal of Engineering in Medicine: 0954411920911277.

Moon, Dae-Hwan, Seung-Hyun Shin, Jong-Beom Na, and Seog-Young Han. 2020. 'Fluid-Structure Interaction Based on Meshless Local Petrov-Galerkin Method for Worm Soft Robot Analysis', International Journal of Precision Engineering and Manufacturing-Green Technology: 1-16.

Mooney, Melvin. 1940. 'A theory of large elastic deformation', Journal of applied physics, 11: 582-92.

Morrow, John, Samantha Hemleben, and Yigit Menguc. 2016. 'Directly fabricating soft robotic actuators with an open-source 3-D printer', IEEE Robotics and Automation Letters, 2: 277-81.

Mosadegh, Bobak, Panagiotis Polygerinos, Christoph Keplinger, Sophia Wennstedt, Robert F Shepherd, Unmukt Gupta, Jongmin Shim, Katia Bertoldi, Conor J Walsh, and George M Whitesides. 2014. 'Pneumatic networks for soft robotics that actuate rapidly', Advanced functional materials, 24: 2163-70.

Moseley, Philip, Juan Manuel Florez, Harshal Arun Sonar, Gunjan Agarwal, William Curtin, and Jamie Paik. 2016. 'Modeling, design, and development of soft pneumatic actuators with finite element method', Advanced engineering materials, 18: 978-88.

Mousavi, Saeb, David Howard, Shuying Wu, and Chun Wang. 2018. 'An Ultrasensitive 3D Printed Tactile Sensor for Soft Robotics', J arXiv preprint arXiv:.09236.

Mustaza, Seri Mastura, Yahya Elsayed, Constantina Lekakou, Chakravarthini Saaj, and Jan Fras. 2019. 'Dynamic modeling of fiber-reinforced soft manipulator: A visco-hyperelastic material-based continuum mechanics approach', Soft robotics, 6: 305-17.

Nasiri, Sara, and Mohammad Reza Khosravani. 2020. 'Progress and challenges in fabrication of wearable sensors for health monitoring', Sensors and Actuators A: Physical: 112105. 
Ng, Wei Long, Alvin Chan, Yew Soon Ong, and Chee Kai Chua. 2020. 'Deep learning for fabrication and maturation of 3D bioprinted tissues and organs', Virtual and Physical Prototyping: 1-19.

Nguyen, Anh Minh, and Arthur Seibel. 2019. "Recycling-oriented design in soft robotics." In Actuators, 62. Multidisciplinary Digital Publishing Institute.

Ogden, Raymond William. 1972. 'Large deformation isotropic elasticity-on the correlation of theory and experiment for incompressible rubberlike solids', Proceedings of the Royal Society of London. A. Mathematical and Physical Sciences, 326: 565-84.

Ou, Jifei, Gershon Dublon, Chin-Yi Cheng, Felix Heibeck, Karl Willis, and Hiroshi Ishii. 2016. "Cilllia: 3D printed micro-pillar structures for surface texture, actuation and sensing." In Proceedings of the 2016 CHI Conference on Human Factors in Computing Systems, 5753-64. ACM.

Ozel, Selim, Nehir A Keskin, Darien Khea, and Cagdas D Onal. 2015. 'A precise embedded curvature sensor module for soft-bodied robots', Sensors and Actuators A: Physical, 236: 349-56.

Ozel, Selim, Erik H Skorina, Ming Luo, Weijia Tao, Fuchen Chen, Yixiao Pan, and Cagdas D Onal. 2016. "A composite soft bending actuation module with integrated curvature sensing." In 2016 IEEE International Conference on Robotics and Automation (ICRA), 4963-68. IEEE.

Patel, Dinesh K, Amir Hosein Sakhaei, Michael Layani, Biao Zhang, Qi Ge, and Shlomo Magdassi. 2017. 'Highly stretchable and UV curable elastomers for digital light processing based 3D printing', Advanced Materials, 29: 1606000.

Peele, Bryan N, Thomas J Wallin, Huichan Zhao, and Robert F Shepherd. 2015. '3D printing antagonistic systems of artificial muscle using projection stereolithography', Bioinspiration \& biomimetics, 10: 055003.

Plott, Jeffrey, and Albert Shih. 2017. 'The extrusion-based additive manufacturing of moisture-cured silicone elastomer with minimal void for pneumatic actuators', Additive Manufacturing, 17: 1-14.

Polygerinos, Panagiotis, Zheng Wang, Johannes TB Overvelde, Kevin C Galloway, Robert J Wood, Katia Bertoldi, and Conor J Walsh. 2015. 'Modeling of soft fiber-reinforced bending actuators', IEEE Transactions on Robotics, 31: 778-89.

Ponte Castaneda, P. 1989. 'The overall constitutive behaviour of nonlinearly elastic composites', Proceedings of the Royal Society of London. A. Mathematical and Physical Sciences, 422: 147-71.

Raman, Ritu, Caroline Cvetkovic, and Rashid Bashir. 2017. 'A modular approach to the design, fabrication, and characterization of muscle-powered biological machines', J nature protocols, 12: 519.

Rivlin, RS. 1948. 'Large elastic deformations of isotropic materials IV. Further developments of the general theory', Philosophical Transactions of the Royal Society of London. Series A, Mathematical and Physical Sciences, 241: 379-97.

Roach, Devin J, Xiao Kuang, Chao Yuan, Kaijuan Chen, and H Jerry Qi. 2018. 'Novel ink for ambient condition printing of liquid crystal elastomers for 4D printing', Smart Materials and Structures, 27: 125011.

Robertson, Matthew A, and Jamie Paik. 2017. 'New soft robots really suck: Vacuum-powered systems empower diverse capabilities', Science Robotics, 2: eaan6357.

Robinson, Sanlin S, Kevin W O'Brien, Huichan Zhao, Bryan N Peele, Chris M Larson, Benjamin C Mac Murray, Ilse M Van Meerbeek, Simon N Dunham, and Robert F Shepherd. 2015. 'Integrated soft sensors and elastomeric actuators for tactile machines with kinesthetic sense', Extreme Mechanics Letters, 5: 47-53.

Rosalia, Luca, Benjamin Wee-Keong Ang, and Raye Chen-Hua Yeow. 2018. 'Geometry-based customization of bending modalities for $3 \mathrm{~d}$-printed soft pneumatic actuators', IEEE Robotics and Automation Letters, 3: 3489-96.

Runge, Gundula, Mats Wiese, and Annika Raatz. 2017. "FEM-based training of artificial neural networks for modular soft robots." In 2017 IEEE International Conference on Robotics and Biomimetics (ROBIO), 385-92. IEEE. 
Sankar, Sriramana, Alisa Brown, Darshini Balamurugan, Harrison Nguyen, Mark Iskarous, Talya Simcox, Deepesh Kumar, Andrei Nakagawa, and Nitish Thakor. 2019. "Texture Discrimination using a Flexible Tactile Sensor Array on a Soft Biomimetic Finger." In 18th IEEE Sensors, SENSORS 2019, 8956704. Institute of Electrical and Electronics Engineers Inc.

Schaffner, Manuel, Jakob A Faber, Lucas Pianegonda, Patrick A Rühs, Fergal Coulter, and André R Studart. 2018. '3D printing of robotic soft actuators with programmable bioinspired architectures', Nature communications, 9: 1-9.

Scharff, Rob BN, Eugeni L Doubrovski, Wim A Poelman, Pieter P Jonker, Charlie CL Wang, and Jo MP Geraedts. 2017. 'Towards behavior design of a 3D-printed soft robotic hand.' in, Soft Robotics: Trends, Applications and Challenges (Springer).

Scharff, Rob BN, Jun Wu, Jo MP Geraedts, and Charlie CL Wang. 2019. "Reducing Out-of-Plane Deformation of Soft Robotic Actuators for Stable Grasping." In 2019 2nd IEEE International Conference on Soft Robotics (RoboSoft), 265-70. IEEE.

Shih, Benjamin, Caleb Christianson, Kyle Gillespie, Sebastian Lee, Jason Mayeda, Zhaoyuan Huo, and Michael Thomas Tolley. 2019. 'Design considerations for 3D printed, soft, multimaterial resistive sensors for soft robotics', Frontiers in Robotics and Al, 6: 30.

Shih, Benjamin, Dylan Shah, Jinxing Li, Thomas G Thuruthel, Yong-Lae Park, Fumiya lida, Zhenan Bao, Rebecca Kramer-Bottiglio, and Michael T Tolley. 2020. 'Electronic Skins for Intelligent Soft Robots', Science Robotics, 5.

Sonar, Harshal A, Aaron P Gerratt, Stéphanie P Lacour, and Jamie Paik. 2020. 'Closed-loop haptic feedback control using a self-sensing soft pneumatic actuator skin', Soft robotics, 7: 22-29.

Stalin, Thileepan, Naresh K Thanigaivel, Vincent S Joseph, and P Valdiviay Alvarado. 2019.

"Automated fiber embedding for tailoring mechanical and functional properties of soft robot components." In 2019 2nd IEEE International Conference on Soft Robotics (RoboSoft), 76267. IEEE.

Stano, Gianni, Luca Arleo, and Gianluca Percoco. 2020. 'Additive Manufacturing for Soft Robotics: Design and Fabrication of Airtight, Monolithic Bending PneuNets with Embedded Air Connectors', Micromachines, 11: 485.

Sun, Enlai, Tao Wang, and Shiqiang Zhu. 2020. 'An experimental study of bellows-type fluidic soft bending actuators under external water pressure', Smart Materials and Structures.

Sun, Yi, Yun Seong Song, and Jamie Paik. 2013. "Characterization of silicone rubber based soft pneumatic actuators." In 2013 IEEE/RSJ International Conference on Intelligent Robots and Systems, 4446-53. leee.

Sun, Yilin, Qiuju Zhang, Xiaoyan Chen, and Haiwei Chen. 2019. 'An Optimum Design Method of PneuNet Actuators for Trajectory Matching Utilizing a Bending Model and GA', Mathematical Problems in Engineering, 2019.

Tan, HW, T Tran, and CK Chua. 2016. 'A review of printed passive electronic components through fully additive manufacturing methods', Virtual and Physical Prototyping, 11: 271-88.

Tani, Kosuke, Hiroyuki Nabae, Gen Endo, and Koichi Suzumori. 2020. 'Pneumatic Soft Actuator Using Self-excitation Based on Automatic-Jet-Switching-Structure', IEEE Robotics and Automation Letters.

Tawk, Charbel, Yuan Gao, Rahim Mutlu, and Gursel Alici. 2019. "Fully 3D printed monolithic soft gripper with high conformal grasping capability." In 2019 IEEE/ASME International Conference on Advanced Intelligent Mechatronics (AIM), 1139-44. IEEE.

Tawk, Charbel, Andrew Gillett, Marc in het Panhuis, Geoffrey M Spinks, and Gursel Alici. 2019. 'A 3Dprinted omni-purpose soft gripper', IEEE Transactions on Robotics, 35: 1268-75.

Terryn, Seppe, Joost Brancart, Dirk Lefeber, Guy Van Assche, and Bram Vanderborght. 2017. 'Selfhealing soft pneumatic robots', Sci. Robot, 2: 1-12.

Thrasher, Carl J, Johanna J Schwartz, and Andrew J Boydston. 2017. 'Modular elastomer photoresins for digital light processing additive manufacturing', ACS applied materials \& interfaces, 9: 39708-16. 
Thuruthel, Thomas George, Egidio Falotico, Mariangela Manti, and Cecilia Laschi. 2018. 'Stable open loop control of soft robotic manipulators', IEEE Robotics and Automation Letters, 3: 1292-98.

Thuruthel, Thomas George, Benjamin Shih, Cecilia Laschi, and Michael Thomas Tolley. 2019. 'Soft robot perception using embedded soft sensors and recurrent neural networks', Science Robotics, 4: eaav1488.

Tibbits, Skylar. 2014. '4D printing: multi-material shape change', Architectural Design, 84: 116-21.

Tolley, Michael T, Robert F Shepherd, Bobak Mosadegh, Kevin C Galloway, Michael Wehner, Michael Karpelson, Robert J Wood, and George M Whitesides. 2014. 'A resilient, untethered soft robot', Soft robotics, 1: 213-23.

Truby, Ryan L, Robert K Katzschmann, Jennifer A Lewis, and Daniela Rus. 2019. "Soft Robotic Fingers with Embedded lonogel Sensors and Discrete Actuation Modes for Somatosensitive Manipulation." In 2019 2nd IEEE International Conference on Soft Robotics (RoboSoft), 32229. IEEE.

Truby, Ryan L, Michael Wehner, Abigail K Grosskopf, Daniel M Vogt, Sebastien GM Uzel, Robert J Wood, and Jennifer A Lewis. 2018. 'Soft somatosensitive actuators via embedded 3D printing', Advanced Materials, 30: 1706383.

van Tiem, Joël, Jarno Groenesteijn, Remco Sanders, and Gijs Krijnen. 2015. "3D printed bio-inspired angular acceleration sensor." In SENSORS, 2015 IEEE, 1-4. IEEE.

Walker, James, Thomas Zidek, Cory Harbel, Sanghyun Yoon, F Sterling Strickland, Srinivas Kumar, and Minchul Shin. 2020. "Soft Robotics: A Review of Recent Developments of Pneumatic Soft Actuators." In Actuators, 3. Multidisciplinary Digital Publishing Institute.

Wallin, TJ, J Pikul, and RF Shepherd. 2018. '3D printing of soft robotic systems', J Nature Reviews Materials, 3: 84.

Wallin, TJ, JH Pikul, S Bodkhe, BN Peele, BC Mac Murray, D Therriault, BW McEnerney, RP Dillon, EP Giannelis, and RF Shepherd. 2017. 'Click chemistry stereolithography for soft robots that self-heal', Journal of Materials Chemistry B, 5: 6249-55.

Wang, Hongbo, Greg De Boer, Junwai Kow, Ali Alazmani, Mazdak Ghajari, Robert Hewson, and Peter Culmer. 2016. 'Design methodology for magnetic field-based soft tri-axis tactile sensors', Sensors, 16: 1356.

Wang, Nianfeng, XianDong Ge, Hao Guo, Chaoyu Cui, and Xianmin Zhang. 2017. "A kind of soft pneumatic actuator based on multi-material 3D print technology." In 2017 IEEE International Conference on Robotics and Biomimetics (ROBIO), 823-27. IEEE.

Wang, Qinglei, Xiaohua Zhou, Hongjia Jiang, Guangkai Sun, and Lianqing Zhu. 2019. 'Polyimide sensing layer for bending shape measurement in soft surgical manipulators', Optik, 183: 17988.

Wang, Xiaochen, Tao Geng, Yahya Elsayed, Tommmaso Ranzani, Chakravarthini Saaj, and Constantina Lekakou. 2014. "A new coefficient-adaptive orthonormal basis function model structure for identifying a class of pneumatic soft actuators." In 2014 IEEE/RSJ International Conference on Intelligent Robots and Systems, 530-35. IEEE.

Wang, Zhongkui, Damith Suresh Chathuranga, and Shinichi Hirai. 2016. "3D printed soft gripper for automatic lunch box packing." In 2016 IEEE International Conference on Robotics and Biomimetics (ROBIO), 503-08. IEEE.

Wang, Zhongkui, and Shinichi Hirai. 2016. "A 3D printed soft gripper integrated with curvature sensor for studying soft grasping." In 2016 IEEE/SICE International Symposium on System Integration (SII), 629-33. IEEE.

Wang, Zhongkui, Yuuki Torigoe, and Shinichi Hirai. 2017. 'A prestressed soft gripper: design, modeling, fabrication, and tests for food handling', IEEE Robotics and Automation Letters, 2: 1909-16.

Wang, Zhongkui, Mingzhu Zhu, Sadao Kawamura, and Shinichi Hirai. 2017. 'Comparison of different soft grippers for lunch box packaging', Robotics and biomimetics, 4: 1-9. 
Webster III, Robert J, and Bryan A Jones. 2010. 'Design and kinematic modeling of constant curvature continuum robots: A review', The International Journal of Robotics Research, 29: 1661-83.

Wehner, Michael, Michael T Tolley, Yiğit Mengüç, Yong-Lae Park, Annan Mozeika, Ye Ding, Cagdas Onal, Robert F Shepherd, George M Whitesides, and Robert J Wood. 2014. 'Pneumatic energy sources for autonomous and wearable soft robotics', Soft robotics, 1: 263-74.

White, Edward L, Jennifer C Case, and Rebecca K Kramer. 2017. 'Multi-mode strain and curvature sensors for soft robotic applications', Sensors and Actuators A: Physical, 253: 188-97.

Wickberg, Andreas, Jonathan B Mueller, Yatin J Mange, Joachim Fischer, Thomas Nann, and Martin Wegener. 2015. 'Three-dimensional micro-printing of temperature sensors based on upconversion luminescence', J Applied Physics Letters, 106: 133103.

Wilson, James F. 1984. 'Mechanics of bellows: A critical survey', International Journal of Mechanical Sciences, 26: 593-605.

Wolfer, Tim, Patrick Bollgruen, Dario Mager, Ludger Overmeyer, and Jan G Korvink. 2016. 'Printing and preparation of integrated optical waveguides for optronic sensor networks', J Mechatronics, 34: 119-27.

Yamaguchi, Takafumi, Takayuki Arie, Seiji Akita, and Kuniharu Takei. 2019. "Electronic SkinIntegrated Soft Robotic Hand." In 2019 20th International Conference on Solid-State Sensors, Actuators and Microsystems \& Eurosensors XXXIII (TRANSDUCERS \& EUROSENSORS XXXIII), 543-46. IEEE.

Yamaguchi, Takafumi, Tsutomu Kashiwagi, Takayuki Arie, Seiji Akita, and Kuniharu Takei. 2019. 'Human-Like Electronic Skin-Integrated Soft Robotic Hand', Advanced Intelligent Systems, 1: 1900018.

Yamanaka, Yuta, Sho Katagiri, Hiroyuki Nabae, Koichi Suzumori, and Gen Endo. 2020. "Development of a Food Handling Soft Robot Hand Considering a High-speed Pick-and-place Task." In 2020 IEEE/SICE International Symposium on System Integration (SII), 87-92. IEEE.

Yang, Yang, and Yonghua Chen. 2017. 'Innovative design of embedded pressure and position sensors for soft actuators', IEEE Robotics and Automation Letters, 3: 656-63.

Yap, Hong Kai, Hui Yong Ng, and Chen-Hua Yeow. 2016. 'High-force soft printable pneumatics for soft robotic applications', Soft robotics, 3: 144-58.

Yeoh, Oon H. 1993. 'Some forms of the strain energy function for rubber', Rubber Chemistry and technology, 66: 754-71.

Yoshida, Kazunari, Yuki Takishima, Yuta Hara, Masaru Kawakami, and Hidemitsu Furukawa. 2018. "3D printing for gel robotics." In Nano-, Bio-, Info-Tech Sensors, and 3D Systems II, 1059717. International Society for Optics and Photonics.

Yuan, Xin, Jiakang Zou, Lining Sun, Huicong Liu, and Guoqing Jin. 2019. "Soft tactile sensor and curvature sensor for caterpillar-like soft robot's adaptive motion." In Proceedings of the 2019 International Conference on Robotics, Intelligent Control and Artificial Intelligence, 69095.

Zhang, Ningbin, Lisen Ge, Haipeng Xu, Xiangyang Zhu, and Guoying Gu. 2020. '3D printed, modularized rigid-flexible integrated soft finger actuators for anthropomorphic hands', Sensors and Actuators A: Physical: 112090.

Zhang, Yuan-Fang, Colin Ju-Xiang Ng, Zhe Chen, Wang Zhang, Sahil Panjwani, Kavin Kowsari, Hui Ying Yang, and Qi Ge. 2019. 'Miniature Pneumatic Actuators for Soft Robots by High-Resolution Multimaterial 3D Printing', Advanced Materials Technologies, 4: 1900427.

Zhang, Yuan-Fang, Ningbin Zhang, Hardik Hingorani, Ningyuan Ding, Dong Wang, Chao Yuan, Biao Zhang, Guoying Gu, and Qi Ge. 2019. 'Fast-Response, Stiffness-Tunable Soft Actuator by Hybrid Multimaterial 3D Printing', Advanced Functional Materials, 29: 1806698.

Zhao, Jie, Jun Zhong, and Jizhuang Fan. 2015. 'Position control of a pneumatic muscle actuator using RBF neural network tuned PID controller', Mathematical Problems in Engineering, 2015. 
Zhong, Guoliang, Yangdong Hou, and Weiqiang Dou. 2019. 'A soft pneumatic dexterous gripper with convertible grasping modes', International Journal of Mechanical Sciences, 153: 445-56.

Zhou, Jianshu, Shu Chen, and Zheng Wang. 2017. 'A soft-robotic gripper with enhanced object adaptation and grasping reliability', IEEE Robotics and Automation Letters, 2: 2287-93.

Zhou, Jianshu, Yonghua Chen, Xiaojiao Chen, Zheng Wang, Yunquan Li, and Yunhui Liu. 2020. 'A proprioceptive bellows (PB) actuator with position feedback and force estimation', IEEE Robotics and Automation Letters, 5: 1867-74.

Zhu, Mingzhu, Yoshiki Mori, Tatsuhiro Wakayama, Akira Wada, and Sadao Kawamura. 2019. 'A fully multi-material three-dimensional printed soft gripper with variable stiffness for robust grasping', Soft robotics, 6: 507-19.

Zhu, Mingzhu, Yoshiki Mori, Mengying Xie, Akira Wada, and Sadao Kawamura. 2018. "A 3D Printed Two DOF Soft Robotic Finger With Variable Stiffness." In 2018 12th France-Japan and 10th Europe-Asia Congress on Mechatronics, 387-91. IEEE.

Zhu, Mingzhu, Mengying Xie, Xuanming Lu, Shima Okada, and Sadao Kawamura. 2020. 'A soft robotic finger with self-powered triboelectric curvature sensor based on multi-material 3D printing', Nano Energy: 104772.

Zhuang, Wei, Guangkai Sun, Hong Li, Xiaoping Lou, Mingli Dong, and Lianqing Zhu. 2018. 'FBG based shape sensing of a silicone octopus tentacle model for soft robotics', Optik, 165: 7-15.

Zolfagharian, Ali, Akif Kaynak, Mahdi Bodaghi, Abbas Z Kouzani, Saleh Gharaie, and Saeid Nahavandi. 2020. 'Control-Based 4D Printing: Adaptive 4D-Printed Systems', Applied Sciences, 10: 3020.

Zolfagharian, Ali, Akif Kaynak, and Abbas Kouzani. 2019. 'Closed-loop 4D-printed soft robots', Materials \& Design: 108411.

Zolfagharian, Ali, Abbas Kouzani, Amir Ali Amiri Moghadam, Sui Yang Khoo, Saeid Nahavandi, and Akif Kaynak. 2018. 'Rigid elements dynamics modeling of a 3D printed soft actuator', J Smart Materials Structures, 28: 025003.

Zolfagharian, Ali, Abbas Z Kouzani, Sui Yang Khoo, lan Gibson, and Akif Kaynak. 2016. "3D printed hydrogel soft actuators." In 2016 IEEE Region 10 Conference (TENCON), 2272-77. IEEE.

Zolfagharian, Ali, Abbas Z Kouzani, Sui Yang Khoo, Amir Ali Amiri Moghadam, lan Gibson, and Akif Kaynak. 2016. 'Evolution of 3D printed soft actuators', Sensors and Actuators A: Physical, 250: 258-72.

Zolfagharian, Ali, P Valipour, and SE Ghasemi. 2016. 'Fuzzy force learning controller of flexible wiper system', Neural Computing and Applications, 27: 483-93. 